\title{
A class number formula for higher derivatives of abelian $\boldsymbol{L}$-functions
}

\author{
A. Hayward
}

\begin{abstract}
Gross and Rubin have made conjectures about special values of equivariant $L$-functions associated to abelian extensions of global fields. We describe a common refinement, due to Burns, and give evidence in favour of this conjecture for quadratic extensions and cyclotomic fields. We also note that the statement provides a new interpretation of further conjectures of Darmon and Gross.
\end{abstract}

\section{Introduction}

For $K / k$ a finite abelian extension of global fields with Galois group $G$, and $S$ and $T$ finite disjoint sets of places of $k$ such that $S$ contains all infinite places, one defines an equivariant $L$-function $\Theta_{K / k, S, T}(s)$ for $s \in \mathbb{C}$, valued in $\mathbb{C}[G]$. When $K=k$, this is the ( $S$-truncated, $T$-modified) zeta function of the field $k$. Dirichlet's analytic class number formula tells about the properties of this zeta function at the point $s=0$, specifically its order of vanishing and its leading term. This latter is the product of a transcendental 'regulator' term, formed from the units of $k$, with $h_{k, S, T} / w_{k, S, T}$, a ratio of integer invariants related to the arithmetic in the field $k$.

Towards the end of the 1970s, Stark conjectured analogues of these properties for more general $L$-functions. In particular, for abelian extensions he proposed an integrality statement for $\Theta_{K / k, S, T}^{\prime}(0)$. Of the work which followed this, we note the paper [Rub96] of Rubin, where he made a conjecture which extended Stark's to higher orders of vanishing. Rubin's conjecture has the property that it tends to strengthen as the order of vanishing increases; indeed for the zeroth derivative $\Theta_{K / k, S, T}(0)$, where it states $\Theta_{K / k, S, T}(0) \in \mathbb{Z}[G]$, it follows easily from theorems of Deligne and Ribet (cf. [Rub96, Theorem 3.3]) and Weil [Wei67]. On the other hand, Gross [Gro88] made a conjecture of a different kind for this very element, in which he relates it to the class number $h_{k, S, T}$ and a certain group-ring valued regulator. However, for higher orders of vanishing Gross's conjecture becomes trivial.

In this paper we study a conjecture of Burns (Conjecture 2.6) which unites these two approaches. It represents a strengthening of Rubin's conjecture which is precisely in the spirit of Gross, and it specializes to Gross's conjecture for the zeroth derivative $\Theta_{K / k, S, T}(0)$. The formulation was inspired by work in [Bur01], where it is shown that the Equivariant Tamagawa Number Conjecture, as formulated by Burns and Flach in [BF01], implies, for a certain class of extensions, a stronger variant of Conjecture 2.6. The statement here proposes a generalization of this to arbitrary abelian extensions.

In $\S \S 2-4$, we state the conjecture and give some elementary properties and special cases, including a proof for quadratic extensions. We then go on to use the theory of Dirichlet $L$-functions

Received 24 December 2002, accepted in final form 15 April 2003.

2000 Mathematics Subject Classification 11R42 (primary), 11R29 (secondary).

Keywords: abelian $L$-functions, Rubin-Stark conjecture, Gross's conjecture, cyclotomic fields.

The author was funded during this work by an EPSRC doctoral training grant.

This journal is (C) Foundation Compositio Mathematica 2004. 


\section{A. HAYWARD}

and cyclotomic fields to study the conjecture for certain extensions of number fields. In particular, we give evidence in the case of a real abelian extension of $\mathbb{Q}$.

We also discuss two conjectures bearing a family resemblance to Gross's but concerning 'minus-units' relative to a quadratic extension. These are due to Darmon ([Dar95], dealing with an explicit 'circular unit' related to first derivatives of $L$-functions) and Gross ([Gro88], Conjecture 8.8, which has more general hypotheses and concerns the values of the $L$-functions). In each context we interpret these conjectures as rather striking 'base-change'-type statements for Burns's conjecture, which transport it from an extension $\tilde{L} / k$ to an extension $\tilde{L} K / K$, where $K$ is a quadratic extension of $k$.

\section{Notation and statement}

\subsection{Basic set-up}

Let $F$ be a global field, $S$ a finite nonempty set of places of $F$ containing all the archimedean places.

We define $\mathcal{O}_{F, S}:=\{\alpha \in F: v(\alpha) \geqslant 0$ for all $v \notin S\}$, the ring of $S$-integers of $F$, and $U_{F, S}=\mathcal{O}_{F, S}^{\times}$, the $S$-units. The $S$-class group $A_{F, S}$ is defined to be the Picard group of $\mathcal{O}_{F, S}$, and fits into the exact sequence

$$
0 \longrightarrow U_{F, S} \longrightarrow F^{\times} \longrightarrow \bigoplus_{\mathfrak{p} \notin S} \mathfrak{p}^{\mathbb{Z}} \longrightarrow A_{F, S} \longrightarrow 0 .
$$

Now let $T$ be a finite set of places of $F$, disjoint from $S$. The subgroup of $U_{F, S}$ consisting of those $S$-units congruent to 1 modulo every prime in $T$ is denoted $U_{F, S, T}$. The $S$ ray-class group modulo $T$, denoted $A_{F, S, T}$, is the quotient of the group of fractional ideals of $\mathcal{O}_{F, S}$ prime to $T$ by the subgroup of principal ideals with a generator congruent to 1 modulo each prime in $T$. The class groups fit into an exact sequence

$$
0 \longrightarrow U_{F, S, T} \longrightarrow U_{F, S} \longrightarrow \prod_{\mathfrak{p} \in T} \mathbb{F}_{\mathfrak{p}}^{\times} \longrightarrow A_{F, S, T} \longrightarrow A_{F, S} \longrightarrow 0
$$

where $\mathbb{F}_{\mathfrak{p}}$ denotes the residue field of $F$ at $\mathfrak{p}$. For any finite place $\mathfrak{p}$ of $F$, we let $N_{\mathfrak{p}}$ be the size of $\mathbb{F}_{\mathfrak{p}}$. Define the $S$ - and $(S, T)$-class numbers $h_{F, S}=\# A_{F, S}, h_{F, S, T}=\# A_{F, S, T}$. Then

$$
h_{F, S, T}=h_{F, S} \cdot \frac{\prod_{\mathfrak{p} \in T}(N \mathfrak{p}-1)}{\left(U_{F, S}: U_{F, S, T}\right)}=h_{F, S}\left(\prod_{\mathfrak{p} \in T} \mathbb{F}_{\mathfrak{p}}^{\times}: \widetilde{U_{F, S}}\right),
$$

where $\widetilde{U_{F, S}}$ denotes the image of $U_{F, S}$ in the residue fields.

For the rest of $\S 2$, we fix an abelian extension of global fields $K / k$ with Galois group $G$, and a non-negative integer $r$. Let $S=S_{k}$ and $T=T_{k}$ be finite sets of places of $k$, and define $S_{K}$ and $T_{K}$ to be the sets of places of $K$ dividing places in $S_{k}$ and $T_{k}$, respectively. We will abbreviate $U_{K, S_{K}, T_{K}}$ as $U_{K, S, T}$, and do similarly for the class groups and class numbers. Let $S_{1}$ be a subset of $S$. We assume $S, S_{1}$ and $T$ satisfy the following.

\section{Hypothesis 2.1.}

i) $S$ contains all the archimedean places of $k$;

ii) $S$ contains the places that ramify in $K / k$;

iii) $S_{1}$ consists of $r$ places that split completely in $K / k$;

iv) $\# S \geqslant r+1$;

v) $T \cap S=\emptyset$ and $U_{K, S, T}$ is torsion-free.

We write $\# S=r+d+1$, so $U_{k, S, T}$ is a free abelian group of rank $r+d$. Note that our set-up closely follows [Rub96]. 


\subsection{The equivariant $L$-function}

For a finite unramified place $v$ of $k$ let Frob $v$ be the (arithmetic) Frobenius of the residue extension corresponding to $w / v$ for a place $w$ of $K$ dividing $v$. As $K / k$ is abelian, this is a well-defined element of $G$.

For a character $\chi$ of the Galois group $G$ of the extension $K / k$, write

$$
e_{\chi}(K / k):=\frac{1}{\# G} \sum_{g \in G} \chi(g) g^{-1}
$$

for the corresponding idempotent in $\mathbb{C}[G]$. Define the $S$-truncated abelian (Artin) $L$-function of $\chi$ by

$$
L_{K / k, S}(s, \chi)=\prod_{v \notin S_{k}}\left(1-\chi\left(\operatorname{Frob}_{v}\right) N_{v}^{-s}\right)^{-1} .
$$

The product converges for $\operatorname{Re} s>1$ and it is well known that the function can be meromorphically extended to all of $\mathbb{C}$. The $L$-functions combine to give the $S$-truncated, $T$-modified equivariant $L$-function $\mathbb{C} \longrightarrow \mathbb{C}[G]$, as defined, for example, in [Tat84, ch. IV,$\S 1$ ]:

$$
\begin{aligned}
\Theta_{K / k, S, T}(s) & :=\left(\prod_{t \in T_{k}}\left(1-N_{t}^{1-s} \operatorname{Frob}_{t}^{-1}\right)\right) \sum_{\chi \in \hat{G}} L_{K / k, S_{k}}\left(s, \chi^{-1}\right) e_{\chi}(K / k) \\
& =\left(\prod_{t \in T_{k}}\left(1-N_{t}^{1-s} \operatorname{Frob}_{t}^{-1}\right)\right)\left(\prod_{v \notin S_{k}}\left(1-N_{v}^{-s} \operatorname{Frob}_{v}^{-1}\right)\right)^{-1} .
\end{aligned}
$$

Owing to the assumption that $r$ places in $S_{k}$ split completely in $K / k$, we see by Proposition I.3.4 of [Tat84] that each $L_{K / k, S_{k}}\left(s, \chi^{-1}\right)$ vanishes to order at least $r$ at $s=0$. Write $e_{r}=\sum_{\chi} e_{\chi}$, where the sum is over all those characters for which the order of vanishing is exactly $r$. The $r$ th term in the Taylor expansion is given by

$$
\Theta_{K / k, S, T}^{r}(0):=\lim _{s \rightarrow 0} s^{-r} \Theta_{K / k, S, T}(s) .
$$

It satisfies $\Theta_{K / k, S, T}^{r}(0)=\Theta_{K / k, S, T}^{r}(0) e_{r}$.

\subsection{Special values and units}

We set $Y_{S_{K}}:=\left\{\sum_{v \in S_{K}} n_{v} v: n_{v} \in \mathbb{Z}\right\}$, the free abelian group on $S_{K}$, and $X_{S_{K}}:=\left\{\sum_{v \in S_{K}} n_{v} v \in\right.$ $\left.Y_{S_{K}}: \sum_{v \in S_{K}} n_{v}=0\right\}$ its augmentation subgroup.

Define absolute values at places $v$ of $K$ as follows:

$$
|a|_{v}= \begin{cases}|a| & \text { if } K_{v}=\mathbb{R} \\ |a|^{2} & \text { if } K_{v}=\mathbb{C} \\ N_{v}^{-v(a)} & \text { for } v \text { a finite place }\end{cases}
$$

where the valuation $v$ is normalized so that its image is $\mathbb{Z}$.

For any $\mathbb{Z}[G]$-module $M$ and any ring $R, R M:=R \otimes_{\mathbb{Z}} M$ will denote the $R[G]$-module obtained from $M$ by extending scalars to $R$. The logarithmic regulator map is defined by

$$
\begin{aligned}
\lambda_{S_{K}}: U_{K, S} & \longrightarrow \mathbb{R} X_{S_{K}} \\
u & \longmapsto-\sum_{v \in S_{K}} \ln |u|_{v} v .
\end{aligned}
$$

It is well known that this induces an $\mathbb{R}[G]$-module isomorphism $\mathbb{R} U_{K, S} \rightarrow \mathbb{R} X_{S_{K}}$. Its extension to a map $\bigwedge_{G}^{n} U_{K, S} \longrightarrow \mathbb{R} \bigwedge_{G}^{n} X_{S_{K}}$ will be written as $\lambda_{S_{K}}^{(n)}$. 


\section{A. HAYWARD}

Let us recall the analytic class number formula of Dirichlet. $U_{k, S, T}$ has $\mathbb{Z}$-rank $r+d$, the same as $X_{S_{k}}$. Choose a basis $u_{1}, \ldots, u_{r+d}$ for $U_{k, S, T}$ modulo torsion. Order the elements of $S_{k}$ as $v_{1}, \ldots, v_{r+d+1}$; then $v_{1}-v_{r+d+1}, \ldots, v_{r+d}-v_{r+d+1}$ is a basis for $X_{S_{k}}$. The map $\lambda_{S_{k}}^{(r+d)}$ gives us a real determinant with respect to these bases. The determinant may be calculated as

$$
R_{k, S, T}=\operatorname{det}\left(-\ln \left|u_{i}\right|_{v_{j}}\right)_{1 \leqslant i, j \leqslant r+d} .
$$

The choice of the ordering of $S_{k}$ only affects the determinant up to sign. In this paper we will choose to ignore systematically all questions related to signs of regulators.

Dirichlet's analytic class number formula (see [Gro88, Equation (1.6)]) states that the meromorphic function $\zeta_{k, S, T}$ has a zero of exact order $\# S_{k}-1$ at 0 , and that the coefficient of the leading term in the Taylor expansion here is

$$
-\frac{h_{k, S, T}\left|R_{k, S, T}\right|}{\#\left(U_{k, S, T}\right)_{\text {tors }}}
$$

We now relate $\Theta_{K / k, S, T}^{r}(0)$ to the $S$-units of $K$. Let $W$ be an $r$-tuple $\left(w_{1}, \ldots, w_{r}\right)$ where $w_{i}$ is a place of $K$ chosen above $v_{i} \in S_{1, k}$. Define $w_{i}^{*} \in \operatorname{Hom}_{G}\left(Y_{S_{K}}, \mathbb{Z}[G]\right)$ on $w^{\prime} \in S_{K}$ by $w_{i}^{*}\left(w^{\prime}\right)=\sum_{\gamma} \gamma$, summed over the elements $\gamma$ of $G$ with $\gamma w_{i}=w^{\prime}$. Set $W^{*}=w_{1}^{*} \wedge \cdots \wedge w_{r}^{*} \in \bigwedge_{G}^{r} \operatorname{Hom}_{G}\left(Y_{S_{K}}, \mathbb{Z}[G]\right)$. Then Remark 2 of [Pop99, $\S 1.6]$ shows that

$$
W^{*} \circ \lambda_{S_{K}}^{(r)}:\left(\mathbb{C} \bigwedge_{G}^{r} U_{K, S, T}\right) e_{r} \longrightarrow \mathbb{C}[G] e_{r}
$$

is a $\mathbb{C}[G]$-isomorphism. Hence there is a unique element ${ }^{1}$

$$
\eta=\eta_{K / k, S, T, r, W} \in \mathbb{C} \bigwedge_{G}^{r} U_{K, S, T} e_{r}
$$

such that $W^{*} \circ \lambda_{S_{K}}^{(r)}(\eta)=\Theta_{K / k, S, T}^{r}(0)$. If we choose another place $w \in S_{K}-S_{1, K}$ and set $\mathbf{b}:=$ $\left(w_{1}-w\right) \wedge \cdots \wedge\left(w_{r}-w\right)$, then we have

$$
\Theta_{K / k, S, T}^{r}(0) \bigwedge_{G}^{r} X_{S_{K}}=\Theta_{K / k, S, T}^{r}(0) \mathbb{Z}[G] \mathbf{b}, \quad \lambda_{S_{K}}^{(r)}(\eta)=\Theta_{K / k, S, T}^{r}(0) \mathbf{b} .
$$

We refer to [Rub96], Lemma 2.6(ii) and the proof of Proposition 2.4, for the proof of this.

We are interested in integrality properties of this $\eta_{K / k, S, T, r, W}$, which we will test using elements $\Phi \in \bigwedge_{G}^{r} \operatorname{Hom}_{\mathbb{Z}[G]}\left(U_{K, S, T}, \mathbb{Z}[G]\right)$. It will always suffice for our purposes to assume $\Phi$ is a primitive tensor $\phi_{1} \wedge \cdots \wedge \phi_{r}$ (or $1 \in \mathbb{Z}[G]$ if $r=0$ ), by the linearity of our statements. Then $\Phi\left(u_{1} \wedge \cdots \wedge u_{r}\right)$ means $\operatorname{det}\left(\phi_{j}\left(u_{i}\right)\right)_{i, j}$. The element $\Phi$ induces a $\mathbb{C}$-linear map $\mathbb{C} \bigwedge_{G}^{r} U_{K, S, T} \longrightarrow \mathbb{C}[G]$, and we consider $\Phi(\eta) \in \mathbb{C}[G]$. We propose to strengthen the following conjecture, which is Conjecture $\mathrm{B}^{\prime}$ of $[$ Rub96]:

Conjecture 2.2 (Rubin). For every $\Phi \in \bigwedge_{G}^{r} \operatorname{Hom}_{\mathbb{Z}[G]}\left(U_{K, S, T}, \mathbb{Z}[G]\right)$, we have $\Phi(\eta) \in \mathbb{Z}[G]$.

\subsection{Formulation of the conjecture}

Let aug : $\mathbb{Z}[G] \longrightarrow \mathbb{Z}$ be the augmentation homomorphism, and write $I_{G}$ for its kernel, the augmentation ideal of $\mathbb{Z}[G]$. Assume Rubin's conjecture holds. Burns's conjecture puts further conditions on the group ring element $\Phi(\eta)$, by proposing a congruence for $\Phi(\eta)$ modulo $I_{G}^{d+1}$.

For $G$ any abelian group and $M, N$ any $\mathbb{Z}[G]$-modules, one may make the group $\operatorname{Hom}_{G}(M, N):=$ $\operatorname{Hom}_{\mathbb{Z}[G]}(M, N)$ into a $\mathbb{Z}[G]$-module with the $G$-action given by $(g \alpha)(m)=g \alpha(m)$ for $g \in G$,

\footnotetext{
${ }^{1}$ Rubin [Rub96] denotes this by $\varepsilon$ instead of $\eta$. Throughout, we will omit subscripts from $\eta$ which are clear from the
} context. 
$\alpha \in \operatorname{Hom}_{G}(M, N), m \in M$. There is a canonical isomorphism of abelian groups

$$
\begin{aligned}
\operatorname{Hom}_{\mathbb{Z}}(M, \mathbb{Z}) & \longrightarrow \operatorname{Hom}_{G}(M, \mathbb{Z}[G]) \\
\phi^{1} & \longmapsto\left(x \mapsto \sum_{g \in G} \phi^{1}\left(g^{-1} x\right) g\right) .
\end{aligned}
$$

We write $\phi \mapsto \phi^{1}$ for the inverse of this isomorphism.

In $\S 1.2$ of [Rub96], Rubin observes that for any $\mathbb{Z}$-module $M$ and any $n>0$, every $h \in$ $\operatorname{Hom}_{\mathbb{Z}}(M, \mathbb{Z})$ induces a homomorphism

$$
\begin{gathered}
\tilde{h}: \bigwedge_{\mathbb{Z}}^{n} M \longrightarrow \bigwedge_{\mathbb{Z}}^{n-1} M \\
m_{1} \wedge \cdots \wedge m_{n} \longmapsto \sum_{i=1}^{n}(-1)^{i+1} h\left(m_{i}\right) m_{1} \wedge \cdots \wedge \hat{m}_{i} \wedge \cdots \wedge m_{r}
\end{gathered}
$$

where ' ' ' means 'omit'. This construction can be iterated to obtain

$$
\begin{aligned}
& \bigwedge^{r} \operatorname{Hom}_{\mathbb{Z}}(M, \mathbb{Z}) \longrightarrow \operatorname{Hom}_{\mathbb{Z}}\left(\bigwedge^{n} M, \bigwedge^{n-r} M\right) \\
& h_{1} \wedge \cdots \wedge h_{n} \longmapsto \tilde{h_{1}} \circ \cdots \circ \tilde{h_{n}} .
\end{aligned}
$$

If $\Phi=\phi_{1} \wedge \cdots \wedge \phi_{r} \in \bigwedge_{\mathbb{Z}[G]}^{r} \operatorname{Hom}_{\mathbb{Z}[G]}\left(U_{K, S, T}, \mathbb{Z}[G]\right)$, we define $\tilde{\Phi}$ to be the map from $\bigwedge_{\mathbb{Z}}^{r+d} U_{k, S, T}$ to $\bigwedge_{\mathbb{Z}}^{d} U_{k, S, T}$ thus obtained from $\phi_{1}^{1}, \ldots, \phi_{r}^{1}$.

Definition 2.3. Let $r \leqslant n$ be non-negative integers. Define a set of permutations

$$
\left[\begin{array}{l}
n \\
r
\end{array}\right]:=\left\{\begin{array}{ll}
\sigma \in S_{n}: \begin{array}{l}
\sigma(1)<\sigma(2)<\ldots<\sigma(r) \text { and } \\
\sigma(r+1)<\sigma(r+2)<\ldots<\sigma(n)
\end{array}
\end{array}\right\} .
$$

Note that the cardinality of this set is the binomial coefficient $\left(\begin{array}{l}n \\ r\end{array}\right)$. Each element corresponds to choosing a subset of $r$ elements from $\{1, \ldots, n\}$, and associates to it a sign, $\operatorname{sign}(\sigma)$.

LEMMA 2.4. We have the formula

$$
\tilde{\Phi}\left(u_{1} \wedge \cdots \wedge u_{r+d}\right)=\sum_{\sigma \in\left[\begin{array}{c}
r+d \\
r
\end{array}\right]} \operatorname{sign}(\sigma) \operatorname{det}\left(\phi_{j}^{1}\left(u_{\sigma(i)}\right)\right)_{1 \leqslant i, j \leqslant r} u_{\sigma(r+1)} \wedge \cdots \wedge u_{\sigma(r+d)} .
$$

Proof. The proof is routine.

Now, following Gross, we define a group ring-valued regulator. Let the places in $S_{k}-S_{1, k}$ be denoted $v_{1}^{\prime}, \ldots, v_{d+1}^{\prime}$. For each $v_{i}^{\prime}$, local class field theory gives us a local reciprocity map,

$$
f_{v_{i}^{\prime}}: k^{\times} \longrightarrow G
$$

coming from the reciprocity map in the local extension $K_{w} / k_{v_{i}^{\prime}}$ for a place $w$ of $K$ above $v_{i}^{\prime}$. We compose this with the isomorphism

$$
\begin{gathered}
G \longrightarrow I_{G} / I_{G}^{2} \\
g \longmapsto g-1
\end{gathered}
$$

to get a homomorphism to the additive group $I_{G} / I_{G}^{2}$. We now define the Gross-style regulator homomorphism (cf. [Gro88, Equation (2.2)]):

$$
\begin{aligned}
\operatorname{Reg}_{G}=\operatorname{Reg}_{K / k, S, r}^{\left(v_{1}^{\prime}, \ldots, v_{d}^{\prime}\right)}: \bigwedge_{\mathbb{Z}}^{d} U_{k, S, T} \longrightarrow \mathbb{Z}[G] / I_{G}^{d+1} \\
u_{1} \wedge \cdots \wedge u_{d} \longmapsto \operatorname{det}\left(f_{v_{j}^{\prime}}\left(u_{i}\right)-1\right)_{1 \leqslant i, j \leqslant d}
\end{aligned}
$$




\section{A. HAYWARD}

We will vary the subscripts of Reg according to any clarification needed in context. Note that here we have chosen to exclude $v_{d+1}^{\prime}$. So we need the following.

Proposition 2.5. The homomorphism above does not depend on the choice of which of the $v_{i}^{\prime}$ to exclude, or the ordering of the $v_{i}^{\prime}$, up to sign.

Proof. By the product formula of global class field theory, we have $\prod_{v \in S} f_{v}(x)=1$ for all $x \in U_{k, S}$. As $v \in S_{1}$ split completely in $K / k, f_{v}(x)=1$ for these $v$. Hence

$$
\prod_{v^{\prime} \in S-S_{1}} f_{v^{\prime}}(x)=1 \text {. }
$$

Now choose $j \in\{1, \ldots, d\}$. In the determinant $\operatorname{Reg}_{G}\left(u_{1} \wedge \cdots \wedge u_{d}\right)$, adding every other column to the column corresponding to $v_{j}^{\prime}$ and using the product formula shows that the $i$ th entry in column $j$ is congruent to $-\left(f_{v_{d+1}^{\prime}}\left(u_{i}\right)-1\right)\left(\bmod I_{G}^{2}\right)$. So the determinant becomes $-\operatorname{Reg}_{G}\left(u_{1} \wedge \cdots \wedge u_{d}\right)$ calculated with respect to the places $v_{1}^{\prime}, \ldots, v_{j-1}^{\prime}, v_{d+1}^{\prime}, v_{j+1}^{\prime}, \ldots, v_{d}^{\prime}$. Reordering these can only change the sign again.

Let $u_{1}, \ldots, u_{r+d}$ be a $\mathbb{Z}$-basis for $U_{k, S, T}$. We set $\operatorname{Reg}_{G}^{\Phi}=\operatorname{Reg}_{G}\left(\tilde{\Phi}\left(u_{1} \wedge \cdots \wedge u_{r+d}\right)\right)$, and note that this is independent of the choice of basis up to sign. We have by Lemma 2.4 that

$$
\operatorname{Reg}_{G}^{\Phi}=\sum_{\sigma \in\left[\begin{array}{c}
r+d \\
r
\end{array}\right]} \operatorname{sign}(\sigma) \operatorname{det}\left(\phi_{j}^{1}\left(u_{\sigma(i)}\right)\right)_{1 \leqslant i, j \leqslant r} \operatorname{det}\left(f_{v_{j}^{\prime}}\left(u_{\sigma(r+i)}\right)-1\right)_{1 \leqslant i, j \leqslant d},
$$

where $\left[\begin{array}{c}r+d \\ r\end{array}\right]$ was defined in Definition 2.3. The conjecture we will discuss is as follows.

Conjecture 2.6 (Burns). Let $K / k, S \supseteq S_{1}, T, r$ satisfy Hypothesis 2.1. Assume that Rubin's conjecture holds for this data, so that for every $\Phi \in \bigwedge_{\mathbb{Z}[G]}^{r} \operatorname{Hom}_{\mathbb{Z}[G]}\left(U_{K, S, T}, \mathbb{Z}[G]\right)$, we have $\Phi(\eta) \in$ $\mathbb{Z}[G]$. Then this element satisfies

$$
\Phi(\eta) \equiv \pm h_{k, S, T} \operatorname{Reg}_{G}^{\Phi} \quad\left(\bmod I_{G}^{d+1}\right) .
$$

Note that this conjecture implies $\Phi(\eta) \in I_{G}^{d}$, an 'order of vanishing' statement which generalizes [Gro88, Equation (4.2)] (via Proposition 3.9 in $\S 3$ ). For more on the formulation of Conjecture 2.6, including a method for specifying the sign in the congruence, see [Bur03].

\section{Basic properties of the conjecture}

\subsection{Varying the data}

Firstly, we wish to check that Conjecture 2.6 will remain true if we lower the top field $K$. We note a useful result about the unit groups.

Lemma 3.1. For any $K / k, S, T$ such that $K / k$ is Galois and $U_{K, S, T}$ is torsion-free, the quotient $U_{K, S, T} / U_{k, S, T}$ is also torsion-free.

Proof. Suppose $u \in U_{K, S, T}$ is such that $u^{n} \in U_{k, S, T}$ for some $n>0$. This means that for all $\sigma \in \operatorname{Gal}(K / k)$, we have $\left(u^{n}\right)^{\sigma-1}=1$. However, this is $\left(u^{\sigma-1}\right)^{n}$. Hence for all $\sigma \in \operatorname{Gal}(K / k), u^{\sigma-1}$ is a torsion element of $U_{K, S, T}$ and so is 1 . Hence $u \in k$ as required.

Proposition 3.2. Let $L / K / k$ be a tower of finite extensions, with $L / k$ and $K / k$ abelian with groups $\Gamma$ and $G=\Gamma / H$, respectively. If Conjecture 2.6 holds for $L / k, S \supseteq S_{1}, T$ then it holds for $K / k, S \supseteq S_{1}, T$.

Proof. It is clear, using Proposition IV.1.8 of [Tat84], that $\eta_{K / k}=\left(\bigwedge^{r} N_{L / K}\right) \eta_{L / k}$. 


\section{ABELian $L$-FUnCTIONS}

The inclusion $U_{K, S, T} \hookrightarrow U_{L, S, T}$ and the $\mathbb{Z}[G]$-module isomorphism

$$
\begin{aligned}
& \mathbb{Z}[\Gamma]^{H} \stackrel{\sim}{\longrightarrow}[G] \\
& N_{L / K} \longmapsto 1
\end{aligned}
$$

induce a surjective map

$$
\operatorname{Hom}_{\Gamma}\left(U_{L, S, T}, \mathbb{Z}[\Gamma]\right) \longrightarrow \operatorname{Hom}_{G}\left(U_{K, S, T}, \mathbb{Z}[G]\right),
$$

whereby each $\phi$ in the second group can be lifted to a $\hat{\phi}$ in the first in such a way that the projection of $\hat{\phi}(u)$ to $\mathbb{Z}[G]$ is $\phi\left(N_{L / K} u\right)$ for all $u \in U_{L, S, T}$. This follows by applying [Rub96, Proposition 1.1], to the exact sequence of $\mathbb{Z}$-torsion-free $\Gamma$-modules given by Lemma 3.1, and using [Rub96, Diagram (16)].

Take $\Phi \in \bigwedge_{G}^{r} \operatorname{Hom}_{G}\left(U_{K, S, T}, \mathbb{Z}[G]\right)$ and lift it to $\hat{\Phi} \in \bigwedge_{\Gamma}^{r} \operatorname{Hom}_{\Gamma}\left(U_{L, S, T}, \mathbb{Z}[\Gamma]\right)$ componentwise. Now

$$
\hat{\Phi}\left(\eta_{L / k}\right) \equiv \pm h_{k, S, T} \operatorname{Reg}_{\Gamma}(\tilde{\hat{\Phi}}(\mathbf{u})) \quad\left(\bmod I_{\Gamma}^{d+1}\right)
$$

where $\mathbf{u}=u_{1} \wedge \cdots \wedge u_{r+d}, u_{i}$ a $\mathbb{Z}$-basis of $U_{k, S, T}$. Passing to the quotient in this congruence, and noting that $\tilde{\hat{\Phi}}=\tilde{\Phi}$, we get

$$
\Phi\left(\eta_{K / k}\right) \equiv \pm h_{k, S, T} \operatorname{Reg}_{G}(\tilde{\Phi}(\mathbf{u})) \quad\left(\bmod I_{G}^{d+1}\right),
$$

as required.

We now look at enlarging $T$.

Proposition 3.3. Suppose Conjecture 2.6 holds for $K / k, S \supseteq S_{1}, T$, and $v$ is a place of $k$ not in $S$ or $T$. Set $T^{\prime}=T \cup\{v\}$. Then Conjecture 2.6 holds for $K / k, S \supseteq S_{1}, T^{\prime}$.

Proof. The definition of $\Theta_{K / k, S, T}$ shows that $\eta_{T^{\prime}}=\eta_{T}^{1-N_{v}}$ Frob $_{v}^{-1}$.

We will adapt [Pop02], proof of Proposition 5.3.1. Let $\phi_{1}^{\prime}, \ldots, \phi_{r}^{\prime}$ be in $\operatorname{Hom}_{G}\left(U_{K, S, T^{\prime}}, \mathbb{Z}[G]\right)$ and set $\Phi^{\prime}=\phi_{1}^{\prime} \wedge \cdots \wedge \phi_{r}^{\prime}$. Popescu proves that there exist $\phi_{i} \in \operatorname{Hom}_{G}\left(U_{K, S, T}, \mathbb{Z}[G]\right), \alpha_{i} \in \mathbb{Z}[G]$ and $\phi_{0} \in \operatorname{Hom}_{G}\left(U_{K, S, T^{\prime}}, \mathbb{Z}[G]\right)$ such that $\phi_{i}^{\prime}=\phi_{i}+\alpha_{i} \phi_{0}$ for all $i=1, \ldots, r$. Let $\delta_{v}=1-N_{v}$ Frob $_{v}^{-1}$, then it is clear that the map $\delta_{v} \phi_{0}: x \mapsto \phi_{0}\left(\delta_{v} x\right)$ is in $\operatorname{Hom}_{G}\left(U_{K, S, T}, \mathbb{Z}[G]\right)$. Popescu shows that

$$
\Phi^{\prime}\left(\eta_{T^{\prime}}\right)=\Psi\left(\eta_{T}\right)
$$

where $\Psi \in \bigwedge_{G}^{r} \operatorname{Hom}_{G}\left(U_{K, S, T}, \mathbb{Z}[G]\right)$ is given by

$$
\delta_{v}\left(\phi_{1} \wedge \cdots \wedge \phi_{r}\right)+\sum_{i=1}^{r} \alpha_{i} \phi_{1} \wedge \cdots \wedge \phi_{i-1} \wedge \delta_{v} \phi_{0} \wedge \phi_{i+1} \wedge \cdots \wedge \phi_{r} .
$$

Now let $\mathbf{u}=u_{1} \wedge \cdots \wedge u_{r+d}$ be the wedge of a basis of $U_{k, S, T}$ and $\mathbf{u}^{\prime}=u_{1}^{\prime} \wedge \cdots \wedge u_{r+d}^{\prime}$ similarly for $U_{k, S, T^{\prime}}$. We have $\left(U_{k, S, T}: U_{k, S, T^{\prime}}\right) \mathbf{u}=\mathbf{u}^{\prime}$ in $\bigwedge_{\mathbb{Z}}^{r+d} U_{k, S, T}$. Apply $\tilde{\Psi}$ to both sides of this equality. We note that $\left(\delta_{v} \phi_{0}\right)^{1}\left(u_{i}^{\prime}\right)=\operatorname{aug}\left(\delta_{v}\right) \phi_{0}^{1}\left(u_{i}^{\prime}\right)$ since $u_{i}^{\prime} \in U_{k, S, T^{\prime}}$. Note also that aug $\left(\delta_{v}\right)=-\left(N_{v}-1\right)$. By the form of (7), this shows that

$$
\left(U_{k, S, T}: U_{k, S, T^{\prime}}\right) \tilde{\Psi}(\mathbf{u})=-\left(N_{v}-1\right) \widetilde{\Phi}^{\prime}\left(\mathbf{u}^{\prime}\right) \quad \text { in } \bigwedge_{\mathbb{Z}}^{d} U_{k, S, T} .
$$

But $\left(U_{k, S, T}: U_{k, S, T^{\prime}}\right)$ divides $\left(N_{v}-1\right)$. Since the group in which the equality holds is torsion-free, we may cancel $\left(U_{k, S, T}: U_{k, S, T^{\prime}}\right)$ from both sides. Furthermore, by (3)

$$
h_{k, S, T^{\prime}}=\frac{\left(N_{v}-1\right)}{\left(U_{k, S, T}: U_{k, S, T^{\prime}}\right)} h_{k, S, T} .
$$

So we have $h_{k, S, T} \tilde{\Psi}(\mathbf{u})=-h_{k, S, T^{\prime}} \widetilde{\Phi}^{\prime}\left(\mathbf{u}^{\prime}\right)$ in $\bigwedge_{\mathbb{Z}}^{d} U_{k, S, T}$. Applying $\operatorname{Reg}_{G}$ to this and using (6) gives the result. 


\section{A. HAYWARD}

We next look at changing $S$. We will use the following lemma.

Lemma 3.4. Let $k, S, T$ be such that $U_{k, S, T}$ is torsion-free. Suppose $u_{1}, \ldots, u_{n}$ is a $\mathbb{Z}$-basis for $U_{k, S, T}$. Let $v$ be a place of $k$ not in $S$ or $T$. Take $u^{\prime} \in U_{k, S \cup\{v\}, T}$ such that $v\left(u^{\prime}\right)$ is minimal positive. Then $u_{1}, \ldots, u_{n}, u^{\prime}$ is a basis for $U_{k, S \cup\{v\}, T}$.

Proof. Let $u \in U_{k, S \cup\{v\}, T}$. Then there exists $a \in \mathbb{Z}$ such that $v(u)=a v\left(u^{\prime}\right)$. Then $u / u^{\prime a} \in U_{k, S, T}$, so we see $u_{1}, \ldots, u_{n}, u^{\prime}$ generates $U_{k, S \cup\{v\}, T}$. Linear independence follows from considering the valuations at $v$.

LEMma 3.5. Using the notation of the previous lemma, we have

$$
h_{k, S \cup\{v\}, T} \cdot v\left(u^{\prime}\right)=h_{k, S, T} .
$$

Proof. Write $S^{\prime}=S \cup\{v\}$ for short. The result follows from the analytic class number formula as follows. If $n=\# S-1$, we have

$$
\begin{aligned}
\zeta_{k, S^{\prime}, T}(s) & \equiv h_{k, S^{\prime}, T} R_{k, S^{\prime}, T} s^{n+1} \quad\left(\bmod s^{n+2}\right), \\
\zeta_{k, S, T}(s) & \equiv h_{k, S, T} R_{k, S, T} s^{n} \quad\left(\bmod s^{n+1}\right),
\end{aligned}
$$

and the leading terms are related by

$$
h_{k, S^{\prime}, T} R_{k, S^{\prime}, T}=\lim _{s \rightarrow 0} \frac{1-N_{v}^{-s}}{s} h_{k, S, T} R_{k, S, T} .
$$

Hence $h_{k, S^{\prime}, T} R_{k, S^{\prime}, T}=\left(\ln N_{v}\right) h_{k, S, T} R_{k, S, T}$. On the other hand, the definition of the regulator, and the fact that $v\left(u_{i}\right)=0$ for $i=1, \ldots, n$, shows that $R_{k, S^{\prime}, T}=\ln \left|u^{\prime}\right|_{v} R_{k, S, T}=v\left(u^{\prime}\right)\left(\ln N_{v}\right) R_{k, S, T}$. This gives the result.

Proposition 3.6. Let $K / k, S, T, r$ be data satisfying Hypothesis 2.1, and let $v$ be a place of $k$ not in $S$ or $T$ and which splits completely in $K / k$. Set $S^{\prime}=S \cup\{v\}$ and $S_{1}^{\prime}=S_{1} \cup\{v\}$. Suppose Conjecture 2.6 holds for $K / k, S^{\prime} \supseteq S_{1}^{\prime}, T, r+1$. Then Conjecture 2.6 holds for $K / k, S \supseteq$ $S_{1}, T, r$.

Proof. Choose bases as in Lemma 3.4. Note that ' $n$ ' $=r+d$ in the notation of that lemma, and define $u_{r+d+1}=u^{\prime}$. We choose $w$ above $v$ to go into $W$. By [Rub96, Proposition 5.2] we have $\eta_{S}=\check{w}\left(\eta_{S^{\prime}}\right)$, where $\check{w} \in \operatorname{Hom}_{G}\left(U_{K, S^{\prime}, T}, \mathbb{Z}[G]\right)$ is defined by

$$
\check{w}(u)=\sum_{g \in G} w\left(g^{-1} u\right) g .
$$

Take $\Phi \in \bigwedge_{G}^{r} \operatorname{Hom}_{G}\left(U_{K, S, T}, \mathbb{Z}[G]\right)$. The hypothesis that $U_{K, S, T}$ and $U_{K, S^{\prime}, T}$ are torsion-free implies that the map $\operatorname{Hom}_{G}\left(U_{K, S^{\prime}, T}, \mathbb{Z}[G]\right) \rightarrow \operatorname{Hom}_{G}\left(U_{K, S, T}, \mathbb{Z}[G]\right)$ (restriction) is surjective (cf. [Rub96, Proposition 1.1(ii)]). So we may lift $\Phi$ componentwise to $\Phi^{\prime} \in \bigwedge_{G}^{r} \operatorname{Hom}_{G}\left(U_{K, S^{\prime}, T}, \mathbb{Z}[G]\right)$. Then

$$
\Phi\left(\eta_{S}\right)=\left(\Phi^{\prime} \circ \check{w}\right)\left(\eta_{S^{\prime}}\right) \equiv \pm h_{k, S^{\prime}, T} \operatorname{Reg}_{G}\left(\tilde{\Phi}\left(\tilde{\tilde{w}}\left(u_{1} \wedge \cdots \wedge u^{\prime}\right)\right)\right) \quad\left(\bmod I_{G}^{d+1}\right)
$$

and

$$
\tilde{\tilde{w}}\left(u_{1} \wedge \cdots \wedge u^{\prime}\right)=\sum_{i=1}^{r+d+1}(-1)^{i+1} w\left(u_{i}\right) u_{1} \wedge \cdots \wedge u_{i-1} \wedge u_{i+1} \wedge \cdots \wedge u_{r+d+1} .
$$

However, $u_{1}, \ldots, u_{r+d}$ are $S$-units so this collapses to $\pm w\left(u^{\prime}\right) u_{1} \wedge \cdots \wedge u_{r+d}$. Now because $v$ splits completely in $K / k$, we have $w\left(u^{\prime}\right)=v\left(u^{\prime}\right)$. Hence

$$
\Phi\left(\eta_{S}\right) \equiv \pm v\left(u^{\prime}\right) h_{k, S^{\prime}, T} \operatorname{Reg}_{G}\left(\tilde{\Phi}\left(u_{1} \wedge \cdots \wedge u_{r+d}\right)\right) \quad\left(\bmod I_{G}^{d+1}\right),
$$

which by Lemma 3.5 is what we want. 
Proposition 3.7. Suppose Conjecture 2.6 holds for $K / k, S \supseteq S_{1}, T, r$. Let $v$ be a place of $k$ not in $S$ or $T$, and set $S^{\prime}=S \cup\{v\}$. Then Conjecture 2.6 holds for $K / k, S^{\prime} \supseteq S_{1}, T, r$.

Proof. Again we have $n=r+d$ and define $u_{r+d+1}=u^{\prime}$. We note that, because $S$ satisfies Hypothesis 2.1, $v$ is unramified in $K / k$. Therefore the Artin symbol at $v$ can be calculated by

$$
f_{v}(x)=\left(\operatorname{Frob}_{v}\right)^{v(x)}
$$

for all $x$ in $k^{\times}$. The definition of $\Theta_{K / k, S, T}$ shows that $\eta_{S^{\prime}}=\eta_{S}^{1-F r o b} v$. Take $\Phi=\phi_{1} \wedge \cdots \wedge \phi_{r} \in$ $\bigwedge_{G}^{r} \operatorname{Hom}_{\mathbb{Z}[G]}\left(U_{K, S^{\prime}, T}, \mathbb{Z}[G]\right)$, then Conjecture 2.6 for $S^{\prime}$ asks for

$$
\Phi\left(\eta_{S^{\prime}}\right) \equiv \pm h_{k, S^{\prime}, T} \operatorname{Reg}_{G}\left(\tilde{\Phi}\left(u_{1} \wedge \cdots \wedge u_{r+d+1}\right)\right) \quad\left(\bmod I_{G}^{d+2}\right) .
$$

We choose the places $v_{1}, \ldots, v_{d+1}$ appearing in $\operatorname{Reg}_{G}$ by taking the set $S-S_{1}$ of places not designated as splitting, excluding one place, then adding $v=v_{d+1}$. We have ${ }^{2}$

$$
\operatorname{Reg}_{G}\left(\tilde{\Phi}\left(u_{1} \wedge \cdots \wedge u_{r+d+1}\right)\right)=\sum_{\sigma \in\left[\begin{array}{c}
r+d+1 \\
r
\end{array}\right]} \operatorname{sign}(\sigma) \operatorname{det}\left(\phi_{j}^{1}\left(u_{\sigma(i)}\right)\right) \operatorname{det}\left(f_{v_{j}}\left(u_{\sigma(r+i)}\right)-1\right) .
$$

Let us consider two cases of $\sigma$. If $r+d+1 \in\{\sigma(1), \ldots, \sigma(r)\}$ then in the corresponding term, the column in the second determinant corresponding to $v$ is all zeros, since $u_{1}, \ldots, u_{r+d}$ are $S$-units. The other possibility is that $r+d+1=\sigma(r+d+1)$. Then this same column is all zeros apart from the bottom-right entry, which is

$$
f_{v}\left(u_{r+d+1}\right)-1=\left(\operatorname{Frob}_{v}\right)^{v\left(u^{\prime}\right)}-1 \equiv v\left(u^{\prime}\right)\left(\operatorname{Frob}_{v}-1\right) \quad\left(\bmod I_{G}^{2}\right) .
$$

Hence $\operatorname{Reg}_{K / k, S^{\prime}}\left(\tilde{\Phi}\left(u_{1} \wedge \cdots \wedge u_{r+d+1}\right)\right)= \pm v\left(u^{\prime}\right)\left(\operatorname{Frob}_{v}-1\right) \operatorname{Reg}_{K / k, S}\left(\tilde{\Phi}\left(u_{1} \wedge \cdots \wedge u_{r+d}\right)\right)$, where in the second expression we may consider $\Phi$ as being restricted to $U_{K, S, T}$. So using, Lemma 3.5, Conjecture 2.6 for $S^{\prime}$ now reads,

$$
\left(1-\operatorname{Frob}_{v}\right) \Phi\left(\eta_{S}\right) \equiv \pm\left(\operatorname{Frob}_{v}-1\right) h_{k, S, T} \operatorname{Reg}_{G}\left(\tilde{\Phi}\left(u_{1} \wedge \cdots \wedge u_{r+d}\right)\right) \quad\left(\bmod I_{G}^{d+2}\right) .
$$

Therefore if Conjecture 2.6 holds for $S$, it holds for $S^{\prime}$.

On the other hand, the proof shows that we have the following, possibly weaker, implication in the other direction.

Proposition 3.8. Suppose Conjecture 2.6 holds for $K / k, S \cup\{v\} \supseteq S_{1}, T, r$. Then we have, for the data $K / k, S \supseteq S_{1}, T, r$,

$$
\left(1-\operatorname{Frob}_{v}\right) \Phi(\eta) \equiv\left(1-\operatorname{Frob}_{v}\right)\left( \pm h_{k, S, T} \operatorname{Reg}_{G}^{\Phi}\right) \quad\left(\bmod I_{G}^{d+1}\right),
$$

in the notation of Conjecture 2.6. That is, we obtain the image of the congruence in the next level of the augmentation filtration under multiplication by $\left(1-\right.$ Frob $\left._{v}\right)$.

\subsection{Special cases}

We study the behaviour of the conjecture for some interesting special cases of the data.

Proposition 3.9. Suppose $r=0$ and $K / k, S \supseteq \emptyset, T, 0$ satisfies Hypothesis 2.1, that is we designate no places as splitting in $K / k$. Then Conjecture 2.6 is equivalent to Conjecture 4.1 of [Gro88], up to sign.

Proof. The element $\eta \in \mathbb{C} \bigwedge_{G}^{0} U_{K, S, T} e_{r}=\mathbb{C}[G] e_{r}$ is characterized by $\eta=\Theta_{K / k, S, T}(0)$. Taking $\Phi=1 \in \mathbb{Z}[G]$, Conjecture 2.6 now reads

$$
\Theta_{K / k, S, T}(0) \equiv \pm h_{k, S, T} \operatorname{Reg}_{G}\left(u_{1} \wedge \cdots \wedge u_{d}\right) \quad\left(\bmod I_{G}^{d+1}\right) .
$$

This is a sign-indifferent version of Gross's conjecture for the extension $K / k$ and sets $S$ and $T$.

${ }^{2}$ For the definition of $\left[\begin{array}{c}r+d+1 \\ r\end{array}\right]$ see Definition 2.3 . 


\section{A. HAYWARD}

Proposition 3.10. Conjecture 2.6 holds when more than $r$ places in $S$ split completely.

Proof. We adapt the method of [Rub96, Proposition 3.1].

Note that all the $S$-truncated $L$-functions corresponding to non-trivial characters vanish to order greater than $r$ at $s=0$ (see [Tat84, Proposition I.3.4]). If $\# S>r+1$ then this is also true for the trivial character, and so $\eta$ is the identity. On the other hand, if $\# S>r+1$ then our Gross-style regulators in Conjecture 2.6 can be calculated with respect to a totally split place and so are all zero. Hence Conjecture 2.6 says $0 \equiv 0$.

Now assume $\# S=r+1$. Let $u_{1}, \ldots, u_{r}$ be a $\mathbb{Z}$-basis for $U_{k, S, T}$. In his proof of [Rub96, Proposition 3.1], Rubin shows that by the analytic class number formula

$$
\eta=\frac{h_{k, S, T}}{\# G^{r}} u_{1} \wedge \cdots \wedge u_{r}
$$

(for which we might have to invert a unit $u_{1}$ to get the sign right). We apply $\Phi=\phi_{1} \wedge \cdots \wedge \phi_{r} \in$ $\bigwedge_{G}^{r} \operatorname{Hom}_{G}\left(U_{K, S, T}, \mathbb{Z}[G]\right)$ to $\eta$. Note that, because $u_{i} \in k, \phi_{j}\left(u_{i}\right)=\phi_{j}^{1}\left(u_{i}\right) N_{G}$, where $N_{G}=\sum_{g \in G} g$. We obtain

$$
\Phi(\eta)= \pm \frac{h_{k, S, T}}{(\# G)^{r}} N_{G}^{r} \operatorname{det}\left(\phi_{j}^{1}\left(u_{i}\right)\right)= \pm \frac{h_{k, S, T}}{\# G} N_{G} \operatorname{det}\left(\phi_{j}^{1}\left(u_{i}\right)\right) .
$$

Rubin [Rub96] argues by class field theory that $\# G \mid h_{k, S, T}$, so this is an element of $\mathbb{Z}[G]$. Since $N_{G}$ has augmentation $\# G$, reducing this equation $\bmod I_{G}$ gives us Conjecture 2.6.

Corollary 3.11. If $k / k, S, T, r$ satisfy Hypothesis 2.1, then Conjecture 2.6 is true for this data.

Proof. This is because all places in $S$ split completely.

Proposition 3.12. Assume $K / k, S \supseteq S_{1}, T, r$ satisfy Hypothesis 2.1 and furthermore that \# $\geqslant \geqslant$ $r+2$. Assume Conjecture 2.2 holds for this data. Then we have $\Phi(\eta) \in I_{G}$.

Proof. This is [Bur01, Theorem 4.4(iii)]. We reproduce the proof. Since $\zeta_{k, S}$ vanishes to order $r+1$ at $s=0, \Theta_{K / k, S, T}^{r}(0)$ lies in $\mathbb{C} I_{G}$. Hence $N_{K / k} \eta=1$ and so $\Phi(\eta) \in \mathbb{C} I_{G}$. Now if Conjecture 2.2 holds, we have $\Phi(\eta) \in \mathbb{C} I_{G} \cap \mathbb{Z}[G]=I_{G}$, as required.

\section{Quadratic extensions}

In this section we take $K / k, S, T, r$ with $K / k$ quadratic with group $G$ generated by $\tau$. We will assume, using Proposition 3.10, that exactly $r$ places $S_{1}$ split in $K / k$.

Perhaps the most involved arguments of Rubin [Rub96] and Gross [Gro88] are to verify their respective conjectures in this situation. We adapt their methods to prove the following.

Theorem 4.1. Let $K / k, S \supseteq S_{1}, T, r$ be data satisfying Hypothesis 2.1, with $[K: k]=2$. Then Conjecture 2.6 holds.

Remark 4.2. This result provides a new proof of the validity of Gross's conjecture [Gro88, Conjecture 4.1] for quadratic extensions. Its proof avoids the technicalities and special cases considered by Gross in $[$ Gro88, $\S 6]$, using the extra functorial properties of Conjecture 2.6 with respect to an increase in $S$. For comparison, note that $\S 4.2$ corresponds to the known case ' $n=0$ ' of Gross's conjecture [Gro88, Equation (4.3)], and that in $\S 4.3$ the sign of the regulator is irrelevant. 


\subsection{Cohomology of $U_{K, S, T}$}

Let $u_{1}, \ldots, u_{r+d+r}$ be a basis of $U_{K, S, T}$ such that $u_{1}, \ldots, u_{r+d}$ is a basis of $U_{k, S, T}$, which is possible by Lemma 3.1. The relevant structure of this basis is closely related to the Galois cohomology of the $G$-module $U_{K, S, T}$. Our first result in this direction is the following (cf. [Rub96, Theorem 3.5, proof]).

Lemma 4.3. If $H^{1}\left(G, U_{K, S, T}\right) \neq 0$ then we can assume $N_{K / k} u_{r+d+1}=1$.

Proof. Take $u \in U_{K, S, T}$ representing a non-trivial element of $H^{1}\left(G, U_{K, S, T}\right)=U_{K, S, T}^{-} / U_{K, S, T}^{1-\tau}$, where $U_{K, S, T}^{-}$is the set of $(S, T)$-units of norm 1 . Write $u=\epsilon \prod_{i} u_{r+d+i}^{\alpha_{i}}$, where $\epsilon \in U_{k, S, T}$, and write $\epsilon_{i}$ for the norm $u_{r+d+i}^{1+\tau} \in U_{k, S, T}$. Then $u_{r+d+i}^{1-\tau}=u_{r+d+i}^{2} \epsilon_{i}^{-1}$. Therefore we can assume each $\alpha_{i}$ is 0 or 1 . But they cannot all be 0 . Hence $u$ can go into a basis of $U_{K, S, T}$.

Lemma 4.4. We have

$$
\frac{\# \hat{H}^{0}\left(G, U_{K, S, T}\right)}{\# H^{1}\left(G, U_{K, S, T}\right)}=2^{d} .
$$

Proof. Note the left-hand side is the Herbrand quotient $h\left(U_{K, S, T}\right)$ of the $\mathbb{Z}[G]$-module $U_{K, S, T}$ in the sense of $[$ Ser79, ch. VIII, $\S 4]$. The composite isomorphism of $\mathbb{Q}[G]$-modules

$$
\mathbb{Q} U_{K, S, T} \cong \mathbb{Q} X_{S_{K}} \cong \mathbb{Q}[G]^{r} \oplus \mathbb{Q}^{d}
$$

implies that there is an injection of $U_{K, S, T}$ into $\mathbb{Z}[G]^{r} \oplus \mathbb{Z}^{d}$ with finite cokernel. Then $h\left(U_{K, S, T}\right)=$ $h(\mathbb{Z}[G])^{r} h(\mathbb{Z})^{d}=2^{d}$ as required.

Lemma 4.5. If $H^{1}\left(G, U_{K, S, T}\right)=0$, we can assume that $u_{i}=N_{K / k} u_{r+d+i}$ for $i=1, \ldots, r$.

Proof. Write $N u_{r+d+j}=\prod_{i=1}^{r+d} u_{i}^{\alpha_{j i}}$ for $j=1, \ldots, r$. We may perform the following operations on the $r \times(r+d)$ matrix $\left(\alpha_{j i}\right)$ : elementary column operations, which correspond to swapping and multiplying the units in the basis of $U_{k, S, T}$, and elementary row operations, which correspond to swapping and multiplying the units $u_{r+d+1}, \ldots, u_{r+d+r}$.

Thus we can put $\left(\alpha_{j i}\right)$ into diagonal form with integers $a_{1}, \ldots, a_{r}$ on the diagonal. Now suppose some $a_{i}$ is even, so $N u_{r+d+i}=\epsilon^{2}$ for some $\epsilon$ in $U_{k, S, T}$. Then $u_{r+d+i} \epsilon^{-1}$ is a unit in $K-k$ with norm 1. But the group $U_{K, S, T}^{1-\tau} \subseteq U_{k, S, T} U_{K, S, T}^{2}$. So $u_{r+d+i} \epsilon^{-1}$ represents a non-trivial element of

$$
U_{K, S, T}^{-} / U_{K, S, T}^{1-\tau}=H^{1}\left(G, U_{K, S, T}\right) .
$$

This is a contradiction to our assumption. We conclude that each $a_{i}$ is odd. Now replacing $u_{r+d+i}$ by $u_{r+d+i} u_{i}^{-\left[a_{i} / 2\right]}$ gives us the result.

In our situation, noting that $S$ contains a place not splitting in $K$, Lemma 3.4 of [Rub96] states the following.

LEMMA 4.6 (Rubin).

i) $h_{k, S, T} \mid h_{K, S, T}$.

ii) $\# H^{1}\left(G, U_{K, S, T}\right) \mid h_{k, S, T}$.

iii) If $\hat{H}^{0}\left(G, U_{K, S, T}\right)$ and $H^{1}\left(G, U_{K, S, T}\right)$ are trivial then $h_{k, S, T} \equiv h_{K, S, T} / h_{k, S, T}(\bmod 2)$.

We write $\epsilon_{-}=u_{r+d+1} \wedge \cdots \wedge u_{r+d+r}$. In proving his conjecture for quadratic extensions [Rub96, proof of Proposition 3.5], Rubin uses the analytic class number formula to express $\eta$ in terms of this element; we make extensive use of his formulae, which are quoted below. 


\section{A. HAYWARD}

\subsection{The case $d=0$}

We assume $d=0$, that is $\# S=r+1$, and show that the congruence in Conjecture 2.6 holds. We require

$$
\Phi(\eta) \equiv \pm h_{k, S, T}\left|\begin{array}{ccc}
\phi_{1}^{1}\left(u_{1}\right) & \cdots & \phi_{r}^{1}\left(u_{1}\right) \\
\vdots & \ddots & \vdots \\
\phi_{1}^{1}\left(u_{r}\right) & \cdots & \phi_{r}^{1}\left(u_{r}\right)
\end{array}\right| \quad\left(\bmod I_{G}\right)
$$

Note that this is an equality in $\mathbb{Z}[G] / I_{G} \cong \mathbb{Z}$.

Assume first that $H^{1}\left(G, U_{K, S, T}\right)=0$. Then Rubin [Rub96, proof of Proposition 3.5] shows that

$$
\eta= \pm\left(\frac{h_{K, S, T}}{h_{k, S, T}} \frac{(1-\tau)}{2} \pm h_{k, S, T} \frac{(1+\tau)}{2}\right) \epsilon_{-} .
$$

Lemma 4.6 shows the $\mathbb{Q}[G]$-factor here lies in $\mathbb{Z}[G]$, and we note its augmentation is $\pm h_{k, S, T}$. Now we can assume $u_{r+d+i}^{1+\tau}=u_{i}$ for $i=1, \ldots, r$ by Lemma 4.5, so

$$
\phi\left(u_{r+d+i}\right)=\phi^{1}\left(u_{r+d+i}\right)-\tau \phi^{1}\left(u_{r+d+i}\right)+\tau \phi^{1}\left(u_{i}\right) \equiv \phi^{1}\left(u_{i}\right) \quad\left(\bmod I_{G}\right) .
$$

Hence (8) is satisfied in this case.

Now assume $H^{1}\left(G, U_{K, S, T}\right) \neq 0$. Let $\bar{u}_{1}, \ldots, \bar{u}_{r} \in U_{K, S, T}$ such that $N_{K / k} \bar{u}_{i}$ is a basis for $N U_{K, S, T}$. Set $\epsilon_{+}=\bar{u}_{1} \wedge \cdots \wedge \bar{u}_{r}$. Rubin shows (loc. cit.) that

$$
\eta= \pm \frac{h_{k, S, T}}{\# \hat{H}^{0}\left(G, U_{K, S, T}\right)} \frac{(1+\tau)}{2} \epsilon_{+} \pm \frac{h_{K, S, T}}{h_{k, S, T}} \frac{(1-\tau)}{2} \epsilon_{-}
$$

where the $\mathbb{Q}[G]$-factors are again in $\mathbb{Z}[G]$. By Lemma 4.3 we can assume $N_{K / k} u_{r+d+1}=1$, and then

$$
\phi_{j}\left(u_{r+d+1}\right)=\phi_{j}^{1}\left(u_{r+d+1}\right)+\tau \phi_{j}^{1}\left(u_{r+d+1}^{\tau}\right) \equiv \phi_{j}^{1}\left(N_{K / k} u_{r+d+1}\right)=0 \quad\left(\bmod I_{G}\right),
$$

so $\Phi\left(\epsilon_{-}\right) \equiv 0\left(\bmod I_{G}\right)$. On the other hand, $\phi_{j}\left(\bar{u}_{i}\right) \equiv \phi_{j}^{1}\left(N_{K / k} \bar{u}_{i}\right)\left(\bmod I_{G}\right)$ and the index of the group generated by the $N_{K / k} \bar{u}_{i}$ in $U_{k, S, T}$ is $\# \hat{H}^{0}\left(G, U_{K, S, T}\right)$. This shows that (8) also holds in this case.

This verifies Theorem 4.1 in the case $d=0$.

\subsection{The case $d>0$}

Now we assume $d>0$, i.e. $\# S>r+1$. For $d>0, I_{G}^{d} / I_{G}^{d+1}$ is a group of order 2 , so the congruence statement in Conjecture 2.6 only concerns in which power of the augmentation ideal the terms lie. We have $(1-\tau)^{n}=2^{n-1}(1-\tau)$ for $n>0$. Note that the map

$$
\begin{aligned}
I_{G}^{d} / I_{G}^{d+1} & \longrightarrow I_{G}^{d+1} / I_{G}^{d+2} \\
x & \longmapsto(1-\tau) x
\end{aligned}
$$

is a bijection. We have the following freedom to increase $S$.

Lemma 4.7. Let $K / k, S, T, r$ satisfy Hypothesis 2.1 with $K / k$ being a quadratic extension. Assume $d=\# S-r-1>0$. Let $v$ be a place of $k$ not in $S$ or $T$, and set $S^{\prime}=S \cup\{v\}, S_{1}^{\prime}=S_{1} \cup\{v\}$. Then either of the following conditions implies Conjecture 2.6 for $K / k, S, T, r$ :

i) $v$ splits in $K / k$, and Conjecture 2.6 holds for $K / k, S^{\prime} \supseteq S_{1}^{\prime}, T, r+1$;

ii) $v$ is inert in $K / k$, and Conjecture 2.6 holds for $K / k, S^{\prime} \supseteq S_{1}, T, r$.

Proof. This follows from Propositions 3.6 and 3.8, given the structure of the augmentation filtration. 
Rubin shows in [Rub96, proof of Proposition 3.5] that for the case $K / k$ quadratic and $d>0$ we have

$$
\eta= \pm 2 \frac{h_{K, S, T}}{h_{k, S, T}} \frac{(1-\tau)}{2} \epsilon_{-} .
$$

We note that $2^{d-1}(1-\tau) \in I_{G}^{d}$.

It turns out that we only need to consider the congruence statement under the following cohomological assumption. ${ }^{3}$

Lemma 4.8. Suppose $d>0$ and $H^{1}\left(G, U_{K, S, T}\right)=0$. Then the congruence of Conjecture 2.6 is implied by the following statement:

$$
2^{d-1}(1-\tau) \frac{h_{K, S, T}}{h_{k, S, T}} \equiv h_{k, S, T} \operatorname{Reg}_{G}\left(u_{r+1} \wedge \cdots \wedge u_{r+d}\right) \quad\left(\bmod I_{G}^{d+1}\right) .
$$

Proof. We apply Lemma 4.5 to assume that $u_{i}=N_{K / k} u_{r+d+i}$ for $i=1, \ldots r$. Then $\phi_{j}\left(u_{r+d+i}\right) \equiv$ $\phi_{j}^{1}\left(u_{i}\right)\left(\bmod I_{G}\right)$. Thus

$$
\Phi(\eta) \equiv 2^{d-1}(1-\tau) \frac{h_{K, S, T}}{h_{k, S, T}}\left|\begin{array}{ccc}
\phi_{1}^{1}\left(u_{1}\right) & \cdots & \phi_{r}^{1}\left(u_{1}\right) \\
\vdots & \ddots & \vdots \\
\phi_{1}^{1}\left(u_{r}\right) & \cdots & \phi_{r}^{1}\left(u_{r}\right)
\end{array}\right| \quad\left(\bmod I_{G}^{d+1}\right) .
$$

For the right-hand side of the congruence in Conjecture 2.6, we note that if any $u_{i}, 1 \leqslant i \leqslant r$, appears in the argument of $\operatorname{Reg}_{G}$, then the corresponding term is 0 (because $u_{i}$ is a norm from $K$, and therefore in the kernel of all the local reciprocity maps). So the right-hand side collapses to a single term as follows:

$$
h_{k, S, T}\left|\begin{array}{ccc}
\phi_{1}^{1}\left(u_{1}\right) & \cdots & \phi_{r}^{1}\left(u_{1}\right) \\
\vdots & \ddots & \vdots \\
\phi_{1}^{1}\left(u_{r}\right) & \cdots & \phi_{r}^{1}\left(u_{r}\right)
\end{array}\right| \operatorname{Reg}_{G}\left(u_{r+1} \wedge \cdots \wedge u_{r+d}\right) .
$$

This gives the result.

Next we identify a condition for the non-vanishing of the regulator. We need an auxiliary lemma. For Tate's theory of the group cohomology of finite cyclic groups, we refer the reader to [Ser79, ch. VIII, § 4].

Lemma 4.9. Suppose $h_{k, S}=1$. Then there is an isomorphism

$$
\frac{U_{k, S} \cap N K^{\times}}{N U_{K, S}} \cong A_{K, S}^{G}
$$

Proof (cf. [Gro88, p. 191]). We have the exact sequence (from (1))

$$
0 \longrightarrow K^{\times} / U_{K, S} \longrightarrow \bigoplus_{\mathfrak{P} \notin S_{K}} \mathfrak{P}^{\mathbb{Z}} \longrightarrow A_{K, S} \longrightarrow 0 .
$$

Considering the decompositions of primes shows that $H^{1}\left(G, \bigoplus_{\mathfrak{P} \notin S_{K}} \mathfrak{P}^{\mathbb{Z}}\right)=0$. Then taking cohomology gives an exact sequence

$$
0 \longrightarrow H^{0}\left(G, K^{\times} / U_{K, S}\right) \longrightarrow \bigoplus_{\mathfrak{p} \notin S_{k}} \mathfrak{p}^{\mathbb{Z}} \stackrel{0}{\longrightarrow} A_{K, S}^{G} \longrightarrow H^{1}\left(G, K^{\times} / U_{K, S}\right) \longrightarrow 0 .
$$

Observe that $k^{\times} / U_{k, S}$ injects into $H^{0}\left(G, K^{\times} / U_{K, S}\right)$, and (1) for $k$ shows that it surjects onto $\bigoplus_{\mathfrak{p} \notin S_{k}} \mathfrak{p}^{\mathbb{Z}}$, because the $S$-class group of $k$ is trivial. This is why the map marked 0 is zero. Therefore, we have $A_{K, S}^{G} \cong H^{1}\left(G, K^{\times} / U_{K, S}\right)$.

\footnotetext{
${ }^{3}$ In fact it is easy to show that both sides of the congruence vanish if this assumption is not satisfied.
} 


\section{A. HAYWARD}

On the other hand, applying Hilbert's Theorem 90 [Ser79, ch. X, Proposition 2] and Tate cohomology to the short exact sequence

$$
0 \longrightarrow U_{K, S} \longrightarrow K^{\times} \longrightarrow K^{\times} / U_{K, S} \longrightarrow 0
$$

gives an exact sequence

$$
0 \longrightarrow H^{1}\left(G, K^{\times} / U_{K, S}\right) \longrightarrow \hat{H}^{0}\left(G, U_{K, S}\right) \longrightarrow \hat{H}^{0}\left(G, K^{\times}\right) .
$$

Therefore

$$
H^{1}\left(G, K^{\times} / U_{K, S}\right) \cong \operatorname{ker}\left(U_{k, S} / N U_{K, S} \longrightarrow k^{\times} / N K^{\times}\right)=U_{k, S} \cap N K^{\times} / N U_{K, S} .
$$

This completes the proof.

Lemma 4.10. Suppose $h_{K, S, T}=1$. Then $\operatorname{Reg}_{G}\left(u_{r+1} \wedge \cdots \wedge u_{r+d}\right) \not \equiv 0\left(\bmod I_{G}^{d+1}\right)$.

Proof. By equation (3) and Lemma 4.6 part i, we have that $h_{k, S}=1$, and then Corollary 2 of [Rim65] shows that $H^{1}\left(G, U_{K, S}\right)=0$. Therefore, $\hat{H}^{0}\left(G, U_{K, S}\right)$ is a two-torsion group with $2^{d}$ elements by Lemma 4.4 and hence is isomorphic to $(\mathbb{Z} / 2 \mathbb{Z})^{d}$.

Similar to [Gro88, p. 191], we define a homomorphism

$$
f: U_{k, S} \longrightarrow G^{S-S_{1}} \cong(\mathbb{Z} / 2 \mathbb{Z})^{d+1}
$$

by the local reciprocity maps $f_{v}$. Then, by the product formula,

$$
\operatorname{im} f \subseteq V:=\left\{\left(g_{v}\right)_{v \in S-S_{1}}: \prod g_{v}=1\right\} \cong(\mathbb{Z} / 2 \mathbb{Z})^{d} .
$$

Now $u \in U_{k, S}$ is in ker $f$ if and only if $u$ is a local norm at all the places in $S-S_{1}$, and we note it is automatically a norm at all other places. Since $K / k$ is cyclic, $u$ is a local norm everywhere if and only if it is a global norm. So $\operatorname{ker} f=U_{k, S} \cap N K^{\times}$. Since $h_{K, S, T}=1, A_{K, S}^{G}=0$ and so Lemma 4.9 shows that $\operatorname{ker} f=N U_{K, S}$. On the other hand, $\hat{H}^{0}\left(G, U_{K, S}\right)=U_{k, S} / N U_{K, S} \cong(\mathbb{Z} / 2 \mathbb{Z})^{d}$, so we have $f\left(U_{k, S}\right)=V$.

We note that the form of our regulator and the choice of our unit basis show that the nonvanishing of our regulator

$$
\operatorname{Reg}_{G}\left(u_{r+1} \wedge \cdots \wedge u_{r+d}\right) \not \equiv 0 \quad\left(\bmod I_{G}^{d+1}\right)
$$

is equivalent to saying $f\left(U_{k, S, T}\right)=V$.

The reduction map $U_{K, S} \longrightarrow \prod_{\mathfrak{P} \in T_{K}} \mathbb{F}_{\mathfrak{P}}^{\times}$is surjective, by (2) for $K$ and the assumption that $h_{K, S, T}=1$. Also, the norm in an extension of finite fields is surjective. Hence ker $f=N U_{K, S}$ surjects onto $\prod_{\mathfrak{p} \in T} \mathbb{F}_{\mathfrak{p}}^{\times}$. The sequence (2) for $k$ shows that this latter is isomorphic by the reduction map to $U_{k, S} / U_{k, S, T}$. So every element of $U_{k, S}$ can be written as the product of something in ker $f$ by something in $U_{k, S, T}$, which gives our result.

Now consider $A_{K, S, T}$, the $S_{K}$ ray class group modulo $T_{K}$. Let $S_{K}^{\prime}=\left\{w_{1}, \ldots, w_{n}\right\}$ be a set of primes of $\mathcal{O}_{K, S}$ coprime to $T_{K}$ whose classes generate this group. Set $S^{\prime}$ to be the set of places of $k$ lying below these. If $S$ contains $S^{\prime}$, then $h_{K, S, T}=1$. On the other hand, we have the following lemma.

Lemma 4.11. If $h_{K, S, T}=1$, then the congruence of Conjecture 2.6 holds.

Proof. By Lemma 4.6 part i we have that $h_{k, S, T}=1$, which also shows by part ii that $H^{1}\left(G, U_{K, S, T}\right)$ $=0$. So by Lemma 4.8 it is sufficient to show (9) holds. Lemma 4.10 shows that the right-hand side of (9) is not zero. On the other hand $h_{K, S, T} / h_{k, S, T}=1$ so the left-hand side is not zero either. 
Now by Lemma 4.7, we may assume $S^{\prime} \subseteq S$, increasing $r$ by the number of split primes in $S^{\prime}-S$. Then $h_{K, S, T}=1$, so Lemma 4.11 implies that the congruence of Conjecture 2.6 holds.

We have verified Theorem 4.1 in all cases.

\section{Real abelian extensions of $\mathbb{Q}$}

In real abelian extensions of $\mathbb{Q}$, the infinite place splits and the Stark unit is known to be essentially a cyclotomic element. In this section we show that Conjecture 2.6 can be verified (up to a factor of 2 on each side) using the theory of cyclotomic elements.

\subsection{Determination of the special unit}

Suppose $F$ is a totally real, non-trivial, abelian extension of $\mathbb{Q}$ with group $G$ and conductor $m$. We consider Conjecture 2.6 for the extension $F / \mathbb{Q}$. By the Kronecker-Weber theorem, $F$ is contained in $\mathbb{Q}\left(\zeta_{m}\right)$.

We set $S_{\mathbb{Q}}=\{p \mid m\} \cup\{\infty\}, S_{1, \mathbb{Q}}=\{\infty\}$ and $r=1$ in the notation of $\S 2$, noting that the infinite place does indeed split completely in the extension $F / \mathbb{Q}$ because $F$ is real. Let $\infty_{F}$ be the infinite place of $F$ induced by the embedding

$$
\begin{aligned}
\mathbb{Q}\left(\zeta_{m}\right) & \longrightarrow \mathbb{C} \\
\zeta_{m} & \longmapsto e^{2 \pi i / m} .
\end{aligned}
$$

Set $\beta=1-\zeta_{m}$ and, in the notation of $\S 2, W=\left(\infty_{F}\right)$.

Lemma 5.1. $W^{*}\left(\lambda_{S_{F}}\left(N_{\mathbb{Q}\left(\zeta_{m}\right) / F} \beta\right)\right)=2 \Theta_{F / \mathbb{Q}, S_{\mathbb{Q}}, \emptyset}^{1}(0)$.

Proof. We have

$$
W^{*}\left(\lambda_{S_{F}}\left(N_{\mathbb{Q}\left(\zeta_{m}\right) / F} \beta\right)\right)=-\sum_{\sigma \in G} \ln \left|\sigma N_{\mathbb{Q}\left(\zeta_{m}\right) / F} \beta\right| \sigma^{-1} .
$$

On the other hand, the value at $s=0$ of the $L$-function of an even Dirichlet character $\chi$ defined modulo $m$ is given by

$$
L(0, \chi)=0, \quad L^{\prime}(0, \chi)=-\frac{1}{2} \sum_{i=1}^{m-1} \chi(i) \ln \left|1-\zeta_{m}^{i}\right|,
$$

which holds whether or not $m$ is the conductor of $\chi$ (see e.g. [Tat84, $\S$ III.5]). The result follows easily by combining these formulae.

Let $T=T_{\mathbb{Q}}$ be as required by Hypothesis 2.1 , i.e. $T$ contains a prime of odd residue characteristic. Then the $T$-correction factor is

$$
\delta_{T}=\prod_{v \in T}\left(1-N_{v} \operatorname{Frob}_{v}^{-1}\right), \quad \text { i.e. } \Theta_{F / \mathbb{Q}, S, T}^{1}(0)=\delta_{T} \Theta_{F / \mathbb{Q}, S, \emptyset}^{1}(0) .
$$

We have $W^{*}\left(\lambda_{S_{F}}\left(\delta_{T} N_{\mathbb{Q}\left(\zeta_{m}\right) / F} \beta\right)\right)=2 \Theta_{F / \mathbb{Q}, S, T}^{1}(0)$, so (in the notation of $\left.\S 2\right) \delta_{T} N_{\mathbb{Q}\left(\zeta_{m}\right) / F} \beta=2 \eta_{F / \mathbb{Q}}$ in $\mathbb{C} U_{F, S, T}$.

As a result, we wish to study the properties of the cyclotomic elements $\left(1-\zeta_{m}\right)$. The next lemma summarizes their well-known distribution properties.

LEMMA 5.2. For each positive integer $n$, set $\zeta_{n}=e^{2 \pi \mathrm{i} / n}$, and define the norm element of the integral group ring of $\Gamma_{n}:=\operatorname{Gal}\left(\mathbb{Q}\left(\zeta_{n}\right) / \mathbb{Q}\right)$ by $N_{n}:=\sum_{g \in \Gamma_{n}} g$. Take positive integers $p, f, r$ such that $p$ is prime, $f>1$ and $p \nmid f$. By linear disjointness of $\mathbb{Q}\left(\zeta_{p^{r}}\right)$ and $\mathbb{Q}\left(\zeta_{f}\right)$ as extensions of $\mathbb{Q}$, there is a natural inclusion $\Gamma_{p^{r}} \hookrightarrow \Gamma_{p^{r} f}$. Let $\sigma_{a, b}$ denote the automorphism of $\mathbb{Q}\left(\zeta_{b}\right)$ sending $\zeta_{b}$ to $\zeta_{b}^{a}$ for $a$ coprime to $b$. Then we have the following: 


\section{A. HAYWARD}

i) $\left(1-\zeta_{p^{r} f}\right)^{N_{p^{r}}}=\left(1-\zeta_{f}\right)^{\left(1-\sigma_{p, f}^{-1}\right)}$;

ii) $\left(1-\zeta_{p^{r}}\right)^{N_{p^{r}}}=p$;

iii) if two distinct primes divide $n$, then $\left(1-\zeta_{n}\right)^{N_{n}}=1$.

These well-known facts follow from the factorization of $X^{p^{r}}-1 \in \mathbb{C}[X]$.

\subsection{Relations between determinants of certain matrices}

The following linear algebra result will be useful in $\S 5.3$. Fix a commutative ring with 1 , and call it $R$. Let $B$ be a finite set of positive integers, and for each $i, j \in B$ with $i \neq j$ fix $a_{i j} \in R$. For each $I \subseteq B$, let $A^{I}$ be the square matrix indexed by $I$ with $(i, j)$ th entry $a_{i j}$ for $i \neq j$ and $-\sum_{k \in I-\{j\}} a_{i k}$ for $i=j$, so that $A^{I}$ is a matrix with row-sum zero. Let $A_{i}^{I}$ be the $(i, i)$ th minor determinant of $A^{I}$ for $i \in I$.

Proposition 5.3. For each $i \in B$,

$$
\sum_{\{i\} \subseteq I \subseteq B} A_{i}^{I} \prod_{j \in B-I} \sum_{k \in I} a_{j k}=0 .
$$

Proof. The proof uses trees in an analogous way to [GK03, proof of Theorem 8]. If $J$ is a finite set, then a tree $T$ on $J$ consists of the set of vertices $J$ and edges between them which form a connected graph with no loops. A choice of a vertex $r \in J$ to be the 'root' $\sqrt{T}$ of $T$ induces a direction on each edge such that the out-degree of $r$ is 0 and the out-degree of all other vertices is 1 . For a directed tree $T$ on a subset of $B$, define $A(T):=\prod_{(i \rightarrow j) \in T} a_{i j}$.

Since the row-sums of $A^{I}$ are zero, the Kirchhoff-Tutte theorem (see [Tut48] or [GK02, Theorem 4]) states that

$$
A_{i}^{I}=(-1)^{\# I} \sum_{\substack{\text { tree on } I: \\ \sqrt{T}=i}} A(T) .
$$

We also note that

$$
\prod_{j \in B-I} \sum_{k \in I} a_{j k}=\sum_{f:(B-I) \rightarrow I} \prod_{j \in B-I} a_{j, f(j)} .
$$

Hence the left-hand side in the desired equality is

$$
\sum_{\{i\} \subseteq I \subseteq B} \sum_{\substack{T_{I} \\ \text { tree on } I: f_{I}:(B-I) \rightarrow I}}(-1)^{\# I} A\left(T_{I}\right) \prod_{j \in B-I} a_{j, f_{I}(j)} .
$$

For each tree $T$ on $B$ with root $i \in B$, we calculate the coefficient of $A(T)$ in the above sum. If $V$ is a subset of the set of vertices of in-degree 0 in $T$, removing the vertices $V$ and the edges attached to them gives a tree $T_{I}$ on $B-V=: I$. Defining $f_{I}:(B-I) \rightarrow I$ by the relation $\left(j \rightarrow f_{I}(j)\right) \in T$, the pair $(T, V)$ corresponds bijectively to the index $\left(I, T_{I}, f_{I}\right)$ from the sum, whose term is $(-1)^{\# I} A(T)$. But given $T$, there are as many $V$ with \# $I$ even as \#I odd. Hence the term for $T$ is 0 .

\subsection{A congruence statement for cyclotomic elements}

Let $m>1$ and write $m=p_{1}^{a_{1}} p_{2}^{a_{2}} \ldots p_{d+1}^{a_{d+1}}$. Write $\beta_{m}=1-\zeta_{m}$ for the associated cyclotomic element.

If $p \mid m$, and $\mathfrak{p}$ is a place of $\mathbb{Q}\left(\zeta_{m}\right)$ above $p$, we let $f_{p}(x)$ denote the Artin symbol $\left(x, \mathbb{Q}\left(\zeta_{m}\right)_{\mathfrak{p}} / \mathbb{Q}_{p}\right)$ for all non-zero $x \in \mathbb{Z}$. It is a simple exercise in the global class field theory of cyclotomic fields to show the following lemma. 


\section{ABELIAN L-FUnCTIONS}

Lemma 5.4. If $j \neq i, f_{p_{j}}\left(p_{i}\right)^{-1}$ is given by the automorphism $\sigma_{p_{i}, p_{j}^{a_{j}}}$ of $\mathbb{Q}\left(\zeta_{m}\right)$ defined by

$$
\begin{aligned}
\zeta_{p_{j}^{a_{j}}} \mapsto \zeta_{p_{j}}^{p_{i}} \\
\zeta_{p_{k} a_{k}} \mapsto \zeta_{p_{k} a_{k}} \quad \text { for } k \neq j .
\end{aligned}
$$

In the notation of $\S 5.2$, we set $B=\{1, \ldots, d+1\}$ and $a_{i j}=f_{p_{j}}\left(p_{i}\right)-1 \in \mathbb{Z}[\Gamma]$, where $\Gamma:=$ $\operatorname{Gal}\left(\mathbb{Q}\left(\zeta_{m}\right) / \mathbb{Q}\right)$. Then there are defined certain elements $A_{i}^{I} \in \mathbb{Z}[\Gamma]$ for $i \in I \subseteq B$. Set $S=\{p \mid$ $m\} \cup\{\infty\}$, a finite set of places of $\mathbb{Q}$. We prove the following congruence statement for $\beta_{m}$.

Proposition 5.5. For all $\phi: U_{\mathbb{Q}\left(\zeta_{m}\right), S} \longrightarrow \mathbb{Z}[\Gamma]$, we have

$$
\phi\left(\beta_{m}\right) \equiv \sum_{i=1}^{d+1} \phi^{1}\left(p_{i}\right) A_{i}^{B} \quad\left(\bmod I_{\Gamma}^{d+1}\right) .
$$

Proof (cf. [Dar95, Theorem 4.2]). By induction on $d+1$. If $d+1=1$ we have $m=p_{1}^{a_{1}}$. Then

$$
\phi\left(\beta_{m}\right)=\sum_{g \in \Gamma_{m}} \phi^{1}\left(g^{-1} \beta_{m}\right) g \equiv \sum_{g \in \Gamma_{m}} \phi^{1}\left(g^{-1} \beta_{m}\right) \quad\left(\bmod I_{\Gamma}\right),
$$

and this is $\phi^{1}\left(N_{p_{1}^{a_{1}}} \beta_{m}\right)=\phi^{1}\left(p_{1}\right)$ by Lemma 5.2. Hence the claim is true for $d+1=1$.

Now assume it is true for $d+1=1,2, \ldots, n$. Set $d+1=n+1>1$. For $I \subseteq\{1, \ldots, d+1\}$, write $\Gamma_{I}=\prod_{i \in I}\left(\mathbb{Z} / p_{i}^{a_{i}} \mathbb{Z}\right)^{*} \hookrightarrow \Gamma_{m}, \Gamma_{i}=\Gamma_{\{i\}}$ and $m(I)=\prod_{i \in I} p_{i}^{a_{i}}$. We have the following equality in $\mathbb{Z}[\Gamma]:$

$$
\begin{aligned}
& \sum_{g_{1} \in \Gamma_{1}} \ldots \sum_{g_{d+1} \in \Gamma_{d+1}} \phi^{1}\left(\left(g_{1}^{-1} \ldots g_{d+1}^{-1}\right) \beta_{m}\right)\left(g_{1}-1\right) \ldots\left(g_{d+1}-1\right) \\
& \quad=\sum_{g_{1} \in \Gamma_{1}} \ldots \sum_{g_{d+1} \in \Gamma_{d+1}} \phi^{1}\left(\left(g_{1}^{-1} \ldots g_{d+1}^{-1}\right) \beta_{m}\right) \sum_{I \subseteq B}(-1)^{d+1-\# I} \prod_{i \in I} g_{i} \\
& =\sum_{I \subseteq B}(-1)^{d+1-\# I} \sum_{g \in \Gamma_{I}} \phi^{1}\left(g^{-1} \prod_{j \notin I} N_{p_{j}^{a_{j}}} \beta_{m}\right) g .
\end{aligned}
$$

We recall (Lemma 5.2) that, if $I \neq \emptyset$, we have $\prod_{j \notin I} N_{p_{j} a_{j}} \beta_{m}=\prod_{j \notin I}\left(1-\sigma_{p_{j}, m(I)}^{-1}\right) \beta_{m(I)}$, and we note that $\sigma_{p_{j}, m(I)} \in \Gamma_{I}$. For $I=\emptyset, \prod_{j} N_{p_{j} a_{j}} \beta_{m}=1$ by Lemma 5.2. So Equation (11) is equal to

$$
\sum_{\emptyset \neq I \subseteq B}(-1)^{d+1-\# I} \phi_{(I)}\left(\beta_{m(I)}\right) \prod_{j \notin I}\left(1-\sigma_{p_{j}, m(I)}^{-1}\right),
$$

where $\phi_{(I)}$ means the $\mathbb{Z}\left[\Gamma_{I}\right]$-homomorphism $U_{\mathbb{Q}\left(\zeta_{m(I)}\right), S} \longrightarrow \mathbb{Z}\left[\Gamma_{I}\right]$ associated to the restriction of $\phi^{1}$ to $U_{\mathbb{Q}\left(\zeta_{m(I)}\right), S}$.

Lemma 5.4 shows that $\sigma_{p, m(I)}=\prod_{i \in I} f_{p_{i}}(p)^{-1}$ for $p \nmid m(I)$, and our induction hypothesis gives $\phi_{(I)}\left(\beta_{m(I)}\right) \in I_{\Gamma}^{\# I-1}$ for $I \neq B$. So if we reduce our equality modulo $I_{\Gamma}^{d+1}$, we obtain

$$
\begin{aligned}
0 & \equiv \sum_{I \subseteq B}(-1)^{d+1-\# I} \phi_{(I)}\left(\beta_{m(I)}\right) \prod_{j \notin I}\left(-\sum_{k \in I}\left(f_{p_{k}}\left(p_{j}\right)-1\right)\right) \\
& \equiv \sum_{I \subseteq B} \phi_{(I)}\left(\beta_{m(I)}\right) \prod_{j \notin I}\left(\sum_{k \in I} a_{j k}\right) \quad\left(\bmod I_{\Gamma}^{d+1}\right) .
\end{aligned}
$$




\section{A. HAYWARD}

By the induction hypothesis, $\phi_{(I)}\left(\beta_{m(I)}\right) \equiv \sum_{i \in I} \phi^{1}\left(p_{i}\right) A_{i}^{I}\left(\bmod I_{\Gamma}^{\# I}\right)$ if $I \neq B$. Therefore

$$
0 \equiv \phi\left(\beta_{m}\right)+\sum_{i=1}^{d+1} \phi^{1}\left(p_{i}\right) \sum_{\{i\} \subseteq I \subsetneq B} A_{i}^{I} \prod_{j \in B-I}\left(\sum_{k \in I} a_{j k}\right) .
$$

Now Proposition 5.3 shows that the $i$ th term of the sum is $-\phi^{1}\left(p_{i}\right) A_{i}^{B}$, as required.

\subsection{The congruence statement for a real abelian extension of $\mathbb{Q}$}

Let $F / \mathbb{Q}$ be a finite, real, abelian extension. Let $G$ be the Galois group and $m=p_{1}^{a_{1}} \ldots p_{d+1}^{a_{d+1}}$ the conductor of this extension. Recall from $\S 5.1$ that

$$
2 \eta_{F / \mathbb{Q}}=\delta_{T_{\mathbb{Q}}} N_{\mathbb{Q}\left(\zeta_{m}\right) / F} \beta_{m}
$$

where $\beta_{m}=\left(1-\zeta_{m}\right)$. We set $S_{\mathbb{Q}}=S=\{\infty\} \cup\{p \mid m\}, S_{1}=\{\infty\}, r=1$, and $T$ to be as required by Hypothesis 2.1 .

Set $B=\{1, \ldots, d+1\}$, and define $a_{i j} \in \mathbb{Z}\left[\operatorname{Gal}\left(\mathbb{Q}\left(\zeta_{m}\right) / \mathbb{Q}\right)\right]$ as in $\S 5.3$. Then $a_{i j} \mapsto f_{p_{j}}\left(p_{i}\right)-1 \in$ $\mathbb{Z}[G]$ under the natural projection to $\mathbb{Z}[G]$. We relate the projections $\bar{A}_{i}^{I}$ of the $A_{i}^{I}$ determinants from Proposition 5.5 to our group ring-valued regulators. We must choose an ordered set of $d$ places for the purpose of regulator calculation, and we choose $p_{1}, \ldots, p_{d}$.

Proposition 5.6. Let $\sigma \in S_{d+1}$ (the symmetric group on $B$ ) such that $\sigma(1)<\cdots<\sigma(d)$. Then

$$
\operatorname{Reg}_{G}\left(p_{\sigma(1)} \wedge \cdots \wedge p_{\sigma(d)}\right)=\operatorname{sign}(\sigma) \bar{A}_{\sigma(d+1)}^{B} .
$$

Proof. If $d+1=1$ then both sides are the determinants of $0 \times 0$ matrices and so are 1 . So we suppose $d+1>1$. First assume $\sigma=\mathrm{id}$. The regulator is, by the product rule, the determinant of (the projection of)

$$
\left(\begin{array}{cccc}
c_{1} & a_{12} & \cdots & a_{1 d} \\
a_{21} & c_{2} & \cdots & a_{2 d} \\
\vdots & \vdots & \ddots & \vdots \\
a_{d 1} & a_{d 2} & \cdots & c_{d}
\end{array}\right),
$$

with $c_{j}=-\sum_{k} a_{j k}$, where $k$ runs over $\{1, \ldots, d+1\}-\{j\}$. This is what we need.

Now assume $\sigma \neq$ id. Let $b=\sigma(d+1)$. In the matrix defining the regulator, add the other columns to the column for the place $p_{b}$. This gives by the product rule

$$
-\operatorname{Reg}_{G}^{(1,2, \ldots, b-1, n+1, b+1, \ldots, d)}\left(p_{\sigma(1)} \wedge \cdots \wedge p_{\sigma(d)}\right),
$$

where the upper $d$-tuple is the ordered set of places used in the calculation of the regulator. This ordered set differs from the ordered set $(\sigma(1), \ldots, \sigma(d))$ by the permutation $\sigma \circ(n+1 \quad b)$. So

$$
\operatorname{Reg}_{G}^{(1, \ldots, d)}\left(p_{\sigma(1)} \wedge \cdots \wedge p_{\sigma(d)}\right)=\operatorname{sign}(\sigma) \operatorname{Reg}_{G}^{(\sigma(1), \ldots, \sigma(d))}\left(p_{\sigma(1)} \wedge \cdots \wedge p_{\sigma(d)}\right) .
$$

Now the result follows from the case $\sigma=\mathrm{id}$ by renaming the primes $p_{\sigma(i)}$ to $p_{i}$.

Now Proposition 5.5 and a 'lowering the top field' argument entirely analogous to the proof of Proposition 3.2 together prove the following proposition.

Proposition 5.7. For all $\phi: U_{F, S} \longrightarrow \mathbb{Z}[G]$, we have

$$
\phi\left(N_{\mathbb{Q}\left(\zeta_{m}\right) / F} \beta_{m}\right) \equiv(-1)^{d} \sum_{i=1}^{d+1}(-1)^{i+1} \phi^{1}\left(p_{i}\right) \operatorname{Reg}_{G}\left(p_{1} \wedge \cdots \wedge \hat{p}_{i} \wedge \cdots \wedge p_{d+1}\right) \quad\left(\bmod I_{G}^{d+1}\right),
$$

where the superscript ' $\wedge$ ' means omit. 
Remark 5.8. If $F / \mathbb{Q}$ is cyclic of prime-power degree, and all the $p_{i}$ are totally tamely ramified in $F$, then this result can be deduced from Theorem 1 of [GK03].

Finally, we deduce a $T$-modified version. Let $\delta_{T}=\prod_{v \in T}\left(1-N_{v} \operatorname{Frob}_{v}^{-1}\right)$.

Lemma 5.9. Let $u_{1}, \ldots, u_{d+1}$ be a $\mathbb{Z}$-basis for $U_{\mathbb{Q}, S, T}$. Then we have the following equality in $\bigwedge_{\mathbb{Z}}^{d+1} U_{\mathbb{Q}, S}:$

$$
2 u_{1} \wedge \cdots \wedge u_{d+1}=\left(U_{\mathbb{Q}, S}: U_{\mathbb{Q}, S, T}\right) p_{1} \wedge \cdots \wedge p_{d+1} .
$$

Proof. Write $u_{j}= \pm \prod_{i=1}^{d+1} p_{i}^{c_{j i}}$ for $1 \leqslant j \leqslant d+1$ and a $d+1$ square matrix $C=\left(c_{j i}\right)$ over $\mathbb{Z}$. The $d+2$ square matrix of relations between the $d+2$ generators $-1, p_{1}, \ldots, p_{d+1}$ of $U_{\mathbb{Q}, S} / U_{\mathbb{Q}, S, T}$ is of the form

$$
\left(\begin{array}{l|l}
2 & 0 \\
\hline ? & C
\end{array}\right)
$$

where 2 is the top-left entry. Hence the index $\left(U_{\mathbb{Q}, S}: U_{\mathbb{Q}, S, T}\right)=2 \operatorname{det} C$. On the other hand, we have

$$
u_{1} \wedge \cdots \wedge u_{d+1}=(\operatorname{det} C) p_{1} \wedge \cdots \wedge p_{d+1}+X
$$

where $X$ is a sum of terms of the form $(-1) \wedge x$, so that $2 X=0$. Multiplying this by 2 gives the stated result.

Now we can show the following theorem.

Theorem 5.10. Let $F / \mathbb{Q}$ be a real abelian extension with Galois group $G$ and conductor $m, r=1$, $S_{1}=\{\infty\}, S=\{\infty\} \cup\{p \mid m\}, T \nsubseteq\{2\}$ a finite non-empty set of primes of $\mathbb{Q}$ disjoint from $S$. Then $F / \mathbb{Q}, S \supseteq S_{1}, T, r$ satisfies Hypothesis 2.1. In this case the congruence of Conjecture 2.6 is satisfied up to a factor of 2. That is, for all $\phi \in \operatorname{Hom}_{\mathbb{Z}[G]}\left(U_{F, S, T}, \mathbb{Z}[G]\right)$ we have

$$
2 \phi(\eta) \equiv 2\left( \pm h_{\mathbb{Q}, S, T} \operatorname{Reg}_{G}^{\phi}\right) \quad\left(\bmod I_{G}^{d+1}\right) .
$$

Proof. Let $\phi \in \operatorname{Hom}_{\mathbb{Z}[G]}\left(U_{F, S, T}, \mathbb{Z}[G]\right)$. Applying Proposition 5.7 to the map $x \mapsto \phi\left(x^{\delta_{T}}\right)$ and using Lemma 5.9 shows

$$
2 \phi\left(\delta_{T} N_{\mathbb{Q}\left(\zeta_{m}\right) / F} \beta_{m}\right) \equiv 2 \frac{\operatorname{aug}\left(\delta_{T}\right)}{\left(U_{\mathbb{Q}, S}: U_{\mathbb{Q}, S, T}\right)} \operatorname{Reg}_{G}\left(\tilde{\phi}\left(u_{1} \wedge \cdots \wedge u_{d+1}\right)\right) \quad\left(\bmod I_{G}^{d+1}\right) .
$$

Finally, Equation (3) for $\mathbb{Q}$ shows that $h_{\mathbb{Q}, S, T}=(-1)^{\# T} \operatorname{aug}\left(\delta_{T}\right) /\left(U_{\mathbb{Q}, S}: U_{\mathbb{Q}, S, T}\right)$.

Remark 5.11. In particular, if $G$ is of odd order then Conjecture 2.6 holds.

\section{Base change via a conjecture of Darmon}

We now move on to studying what happens when we make a quadratic extension of the base field $k$ in Conjecture 2.6.

\subsection{Quadratic extension of the base field for $L$-functions}

Let $K / k$ and $\tilde{L} / k$ be linearly disjoint finite abelian extensions of global fields. Assume $[K: k]=2$. Write $L=\tilde{L} K$. Hence we have the following diagram of fields:

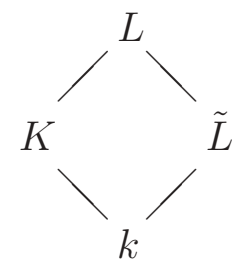




\section{A. HAYWARD}

$L / k$ is Galois with $\operatorname{group} \operatorname{Gal}(L / k)=\operatorname{Gal}(\tilde{L} / k) \times \operatorname{Gal}(K / k)$. Let $\omega$ be the non-trivial character of $\operatorname{Gal}(K / k)$, and let $G:=\operatorname{Gal}(L / K)=\operatorname{Gal}(\tilde{L} / k)$. Let $S=S_{k}$ and $T=T_{k}$ be disjoint finite sets of places of $k$ with $S$ non-empty and containing all infinite places. The Euler factors defining $L$ functions in the extensions $\tilde{L} / k$ and $L / K$ are related as follows. Let $v$ be a finite prime of $k$, then for each place $w$ of $K$ lying over $v$ we have a Frobenius element $\operatorname{Frob}_{w} \in \operatorname{Gal}(L / K) \hookrightarrow \operatorname{Gal}(L / k)$. We compare these with $\operatorname{Frob}_{v} \in \operatorname{Gal}(L / k)$ to obtain

$$
\prod_{\substack{w \text { place of } K \\ w \mid v}}\left(1-N_{w}^{s} \operatorname{Frob}_{w}^{-1}\right)=\left(1-N_{v}^{s} \operatorname{Frob}_{v}^{-1}\right)\left(1-\omega(v) N_{v}^{s} \operatorname{Frob}_{v}^{-1}\right) .
$$

This follows by considering each Euler factor in the three cases $\omega(v)=1(v$ splits in $K / k), \omega(v)=-1$ ( $v$ is inert) and $\omega(v)=0$ ( $v$ ramifies). We see that the $L$-functions satisfy the following base-change factorization when passing from $L / K$ to $L / k$ :

$$
\Theta_{L / K, S_{K}, T_{K}}(s)=\Theta_{L / K / k, S_{k}, T_{k}}(s, \omega) \Theta_{\tilde{L} / k, S_{k}, T_{k}}(s),
$$

where $\Theta_{L / K / k, S, T}(s, \omega)$ is the twisted Stickelberger function defined as

$$
\begin{aligned}
& \left(\prod_{t \in T_{k}}\left(1-\omega(t) N_{t}^{1-s} \operatorname{Frob}_{t}^{-1}\right)\right) \sum_{\chi \in \hat{G}} L_{L / k, S_{k}}\left(s, \omega \chi^{-1}\right) e_{\omega \chi}(L / k) . \\
& =\left(\prod_{t \in T_{k}}\left(1-\omega(t) N_{t}^{1-s} \operatorname{Frob}_{t}^{-1}\right)\right)\left(\prod_{v \notin S_{k}}\left(1-\omega(v) N_{v}^{-s} \operatorname{Frob}_{v}^{-1}\right)\right)^{-1} .
\end{aligned}
$$

The validity of Equation (13) follows from the lemma and definition (4) in the region of convergence $\operatorname{Re} s>1$ and then everywhere by meromorphic continuation.

We will also use the notation

$$
\delta_{T}^{\omega}:=\prod_{v \in T_{k}}\left(1-\omega(v) N_{v} \operatorname{Frob}_{v}^{-1}\right)
$$

for the relative $T$-modification factor at $s=0$.

\subsection{The circular unit}

Here we show how the 'circular unit' defined in [Dar95] corresponds to the change in $L$-functions which results from raising the base field from $\mathbb{Q}$ to a linearly disjoint real quadratic field.

For comparison with [Dar95], we assume the following hypothesis for the rest of $\S 6$.

Hypothesis 6.1 (Darmon's set-up). Let $N$ and $S$ be coprime integers with $N>1$ and $S>1$. Let $\omega$ be a primitive, quadratic, even Dirichlet character defined modulo $N$. Set $K=\mathbb{Q}\left(\zeta_{N}\right)^{\text {ker } \omega}$, a real quadratic field, and call its non-trivial automorphism $\tau$. Let $\tilde{L}$ be a real subfield of $\mathbb{Q}\left(\zeta_{S}\right)$, normal over $\mathbb{Q}$. Write $L=\tilde{L} K$.

Hence we are in the situation of $\S 6.1$ with the further assumptions that $k=\mathbb{Q}$ and that $\tilde{L}$ and $K$ are totally real and have coprime conductors. We define the set $S_{\mathbb{Q}}$ from the integer $S$ in the obvious way: $S_{\mathbb{Q}}=\{p \mid S, \infty\}$.

All the characters of these extensions come from even Dirichlet characters because the fields are totally real. Since, by Equation (10), $L$-functions of even characters vanish at $s=0$, differentiating Equation (13) twice shows that we have the following equality in $\mathbb{C}[G]$

$$
\Theta_{L / K, S_{K}, T_{K}}^{2}(0)=\frac{1}{2} \Theta_{L / K, S_{K}, T_{K}}^{\prime \prime}(0)=\Theta_{L / K / \mathbb{Q}, S_{\mathbb{Q}}, T_{\mathbb{Q}}}^{\prime}(0, \omega) \Theta_{\tilde{L} / \mathbb{Q}, S_{\mathbb{Q}}, T_{\mathbb{Q}}}^{\prime}(0) .
$$


We now relate the base-change factor $\Theta_{L / K / \mathbb{Q}, S_{\mathbb{Q}}, T_{\mathbb{Q}}}^{\prime}(0, \omega)$ to the circular unit defined in $\left[\right.$ Dar95, $\S 4$. This is the following element of $K_{S}:=K\left(\zeta_{S}\right)$ :

$$
\alpha_{S}:=\prod_{\sigma \in \operatorname{Gal}\left(\mathbb{Q}\left(\zeta_{N S}\right) / \mathbb{Q}\left(\zeta_{S}\right)\right)} \sigma\left(\zeta_{N S}-1\right)^{\omega(\sigma)} \in U_{K_{S}} .
$$

Write $\infty_{L}$ for the place of $L$ corresponding to the embedding of $L$ into $\mathbb{R}$ given by $\zeta_{N S} \mapsto e^{2 \pi i / N S}$, and $\overline{\infty_{L}}$ its conjugate by $\tau$.

Lemma 6.2. $\Theta_{L / K / \mathbb{Q}, S_{\mathbb{Q}}, \emptyset}^{\prime}(0, \omega)\left(\infty_{L}-\overline{\infty_{L}}\right)=\frac{1}{2} \lambda_{S_{L}}\left(N_{K_{S} / L} \alpha_{S}\right)$.

Proof. As $N S$ is not a prime power, $\left(\zeta_{N S}-1\right)$ is a global unit in $\mathbb{Q}\left(\zeta_{N S}\right)$. Hence $\lambda_{S_{L}}\left(N_{K_{S} / L} \alpha_{S}\right)$ is zero outside the archimedean places. Now

$$
\lambda_{S_{L}}\left(N_{K_{S} / L} \alpha_{S}\right)=-\sum_{\gamma \in \operatorname{Gal}(L / \mathbb{Q})} \ln \left|\gamma^{-1} N_{K_{S} / L} \alpha_{S}\right| \gamma \infty_{L}=-\sum_{\gamma \in G} \ln \left|\gamma^{-1} N_{K_{S} / L} \alpha_{S}\right| \gamma\left(\infty_{L}-\overline{\infty_{L}}\right),
$$

since the generator of $\operatorname{Gal}(K / \mathbb{Q})$ inverts $\alpha_{S}$. For $\chi$ a character of $L / K$, it suffices to prove

$$
2 \Theta_{L / K / \mathbb{Q}, S_{\mathbb{Q}}, \emptyset}^{\prime}(0, \omega) e_{\omega \chi}(L / \mathbb{Q})=-\sum_{\gamma \in G} \ln \left|\gamma^{-1} N_{K_{S} / L} \alpha_{S}\right| \omega \chi(\gamma) e_{\omega \chi}(L / \mathbb{Q}) .
$$

This is easy to show using (10) for values of the Dirichlet $L$-series at $s=0$, as in Lemma 5.1.

Definition 6.3. We set $\eta_{\omega}:=\delta_{T}^{\omega} N_{K_{S} / L} \alpha_{S}$.

Then $\Theta_{L / K / \mathbb{Q}, S_{\mathbb{Q}}, T_{\mathbb{Q}}}^{\prime}(0, \omega)\left(\infty_{L}-\overline{\infty_{L}}\right)=\frac{1}{2} \lambda_{S_{L}}\left(\eta_{\omega}\right)$.

\subsection{Calculation of $\boldsymbol{\eta}$}

By Hypothesis $6.1, \operatorname{Gal}(L / \mathbb{Q})=\operatorname{Gal}(\tilde{L} / \mathbb{Q}) \times \operatorname{Gal}(K / \mathbb{Q})$ and the restriction map $\operatorname{Gal}(L / K)=G \longrightarrow$ $\operatorname{Gal}(\tilde{L} / \mathbb{Q})$ is an isomorphism. Let $\# S_{\mathrm{s}}=\# S_{\text {split }}$ be the number of primes $p$ dividing $S$ with $\omega(p)=1$, and $\# S_{\mathrm{i}}=\# S_{\text {inert }}$ the number of $p$ with $\omega(p)=-1$.

We consider Conjecture 2.6 for the extensions $L / K$ and $\tilde{L} / \mathbb{Q}$ in turn. We then show that the results and conjecture of [Dar95] relate them.

- $L / K$ : Since $K$ is a real quadratic field, there are two infinite places of $K$, which we call $\infty_{K}$ and $\overline{\infty_{K}}$. As $L$ is also real, these split completely in $L / K$. To avoid confusion, we write $r^{\prime}$ and $d$ ' for ' $r$ ' and ' $d$ ' of $\S 2$ for the extension $L / K$. We take $r^{\prime}=2$ and $S_{1, K}=\left\{\infty_{K}, \overline{\infty_{K}}\right\}$. The element $\eta_{L / K} \in \mathbb{C} \bigwedge_{G}^{2} U_{K, S_{K}, T_{K}} e_{2}$ is defined by

$$
\Theta_{L / K, S_{K}, T_{K}}^{2}(0)\left(\infty_{L}-w_{0}\right) \wedge\left(\overline{\infty_{L}}-w_{0}\right)=\lambda_{S_{L}}^{(2)}\left(\eta_{L / K}\right)
$$

for some finite place $w_{0}$ of $S_{L}$.

- $\tilde{L} / \mathbb{Q}$ : In the notation of $\S 2$ we take $r=1$ and $S_{1, \mathbb{Q}}=\{\infty\}$. Then $d=\# S_{\mathrm{s}}+\# S_{\mathrm{i}}$. The element $\eta_{\tilde{L} / \mathbb{Q}} \in \mathbb{C} U_{\mathbb{Q}, S_{\mathbb{Q}}, T_{\mathbb{Q}}} e_{1}(\tilde{L} / \mathbb{Q})$ is defined by

$$
\Theta_{\tilde{L} / \mathbb{Q}, S_{\mathbb{Q}}, T_{\mathbb{Q}}}^{1}(0)\left(\infty_{\tilde{L}}-v_{0}\right)=\lambda_{S_{\tilde{L}}}\left(\eta_{\tilde{L} / \mathbb{Q}}\right)
$$

for some finite place $v_{0} \in S_{\tilde{L}}$.

The results of $\oint 6.2$ allow us to express $\eta_{L / K}$ in terms of $\eta_{\omega}$ and $\eta_{\tilde{L} / \mathbb{Q}}$. We have

$$
\begin{aligned}
& 2 \Theta_{L / K, S_{K}, T_{K}}^{2}(0)\left(\infty_{L}-w_{0}\right) \wedge\left(\overline{\infty_{L}}-w_{0}\right) \\
& \quad=\Theta_{L / K / \mathbb{Q}, S_{\mathbb{Q}}, T_{\mathbb{Q}}}^{1}(0, \omega)\left(\infty_{L}-\overline{\infty_{L}}\right) \wedge \Theta_{\tilde{L} / \mathbb{Q}, S_{\mathbb{Q}}, T_{\mathbb{Q}}}^{\prime}(0)\left(\infty_{L}+\overline{\infty_{L}}-2 w_{0}\right) .
\end{aligned}
$$




\section{A. HAYWARD}

We now calculate $\lambda_{S_{L}}\left(\eta_{\tilde{L} / \mathbb{Q}}\right)$. Let us review the various identifications and inclusions. Along with the canonical identification of the Galois groups $G=\operatorname{Gal}(\tilde{L} / \mathbb{Q})$, we define the homomorphisms $U_{\tilde{L}} \hookrightarrow U_{L}$ (inclusion) and

$$
\begin{aligned}
i_{L / \tilde{L}}: Y_{S_{\tilde{L}}} & \longmapsto Y_{S_{L}} \\
v & \longmapsto w+\bar{w},
\end{aligned}
$$

where $w$ is a place of $L$ chosen arbitrarily above the place $v$ of $\tilde{L}$, and $\bar{w}=w^{\tau}$. With these maps, the following diagrams commute:

$$
\begin{array}{ccccccc}
\operatorname{Gal}(\tilde{L} / \mathbb{Q}) \times Y_{S_{\tilde{L}}} & \longrightarrow & Y_{S_{\tilde{L}}} & U_{\tilde{L}} & \stackrel{\lambda_{S_{\tilde{L}}}}{\longrightarrow} & \mathbb{R} X_{S_{\tilde{L}}} \\
\downarrow & \square & \downarrow i_{L / \tilde{L}} & \downarrow & \square & \downarrow i_{L / \tilde{L}} \\
G \times Y_{S_{L}} & \longrightarrow & Y_{S_{L}}, & U_{L} & \stackrel{\lambda_{S_{L}}}{\longrightarrow} & \mathbb{R} X_{S_{L}} .
\end{array}
$$

Hence $\lambda_{S_{L}}\left(\eta_{\tilde{L} / \mathbb{Q}}\right)=\Theta_{\tilde{L} / \mathbb{Q}, S_{\mathbb{Q}}, T_{\mathbb{Q}}}^{\prime}(0)\left(\infty_{L}+\overline{\infty_{L}}-w_{1}-\overline{w_{1}}\right)$, where $w_{1}$ is a place of $L$ chosen to be above $v_{0}$. If we assume $\# S_{\mathrm{i}} \neq 0$, we can choose $v_{0}$ such that $w_{1}=\overline{w_{1}}$. Then setting $w_{0}=w_{1}$ in Equation (15) shows that

$$
\lambda_{S_{L}}^{(2)}\left(\eta_{\omega} \wedge \eta_{\tilde{L} / \mathbb{Q}}\right)=4 \Theta_{L / K, S_{K}, T_{K}}^{2}(0)\left(\infty_{L}-w_{0}\right) \wedge\left(\overline{\infty_{L}}-w_{0}\right)
$$

and hence $4 \eta_{L / K}=\eta_{\omega} \wedge \eta_{\tilde{L} / \mathbb{Q}}$.

In [Dar95], Darmon makes a congruence conjecture for his circular unit. We propose to interpret this as a base-change statement for Conjecture 2.6, under the following assumptions.

HYPOTHESIS 6.4.

i) $\# S_{\mathrm{i}} \neq 0$.

ii) For every place $p$ in $T_{\mathbb{Q}}$, we have $\omega(p)=1$.

For a group $U$ on which $\tau$ acts, we define $U^{-}=\left\{u \in U: u^{\tau}=u^{-1}\right\}$.

Proposition 6.5. Assuming Hypothesis 6.4, $\delta_{T}^{\omega}\left(U_{L, S}^{-}\right) \subseteq U_{L, S, T}^{-}$. Therefore $\eta_{\omega} \in U_{L, S, T}^{-}$.

Proof. Let $x \in U_{L, S}^{-}$and $y=x^{\delta_{T}^{\omega}}$. Let $v \in T$ split into $w, \bar{w}$ in $K$. Then $\delta_{T}^{\omega}$ contains a factor $\left(1-N_{w} \operatorname{Frob}_{w}^{-1}\right)$ by Equation $(12)$. Hence $w(y-1)>0$. However, we also have $\bar{w}(y-1)=$ $w\left(y^{-1}-1\right)=w((1-y) / y)=w(1-y)>0$. Therefore $y \equiv 1(\bmod t)$ for all $t \in T_{K}$ as required. Setting $x=N_{K_{S} / L} \alpha_{S}$ proves the second assertion.

\subsection{Indices of minus units}

Let $K / k$ be a quadratic Galois extension of global fields with Galois group generated by $\tau$. For this section we only need to assume that $S$ is a finite, non-empty set of places of $k$ containing all infinite places, and that $T$ is any finite disjoint set of places of $k$.

Lemma 6.6. $\left(U_{K, S}: U_{K, S, T}\right)=\left(U_{k, S}: U_{k, S, T}\right)\left(U_{K, S}^{1-\tau}: U_{K, S, T}^{1-\tau}\right)$.

Proof. Consider the following commutative diagram, in which the rows are exact:

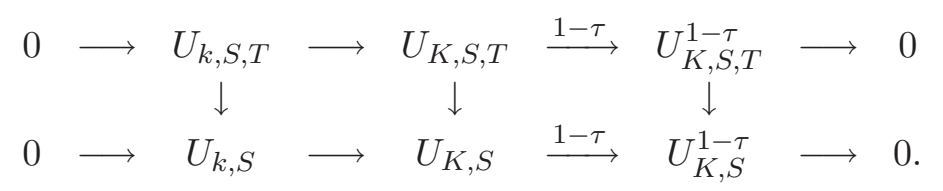

The vertical arrows are inclusions. Applying the snake lemma, we obtain the exact sequence

$$
0 \longrightarrow U_{k, S} / U_{k, S, T} \longrightarrow U_{K, S} / U_{K, S, T} \stackrel{1-\tau}{\longrightarrow} U_{K, S}^{1-\tau} / U_{K, S, T}^{1-\tau} \longrightarrow 0 .
$$

This shows the result. 
We consider the subgroup $U_{K, S, T}^{-}:=\left\{u \in U_{K, S, T}: u^{\tau}=u^{-1}\right\}$ of 'minus $S$-units' in $U_{K, S, T}$. This contains $U_{K, S, T}^{1-\tau}$. The quotient is, by Tate's finite group cohomology [Ser79, ch. VIII],

$$
U_{K, S, T}^{-} / U_{K, S, T}^{1-\tau}=H^{1}\left(\langle\tau\rangle, U_{K, S, T}\right) .
$$

Lemma 6.7. Suppose $h_{k, S}=1$. Then:

i) $H^{1}\left(\langle\tau\rangle, U_{K, S, \emptyset}\right)=0$

ii) $\left(U_{K, S}^{1-\tau}: U_{K, S, T}^{1-\tau}\right)=\left(U_{K, S}^{-}: U_{K, S, T}^{-}\right) \# H^{1}\left(\langle\tau\rangle, U_{K, S, T}\right)$.

Proof. Corollary 2 of [Rim65] shows that $H^{1}\left(\langle\tau\rangle, U_{K, S, \emptyset)}\right.$ embeds into the $S$-class group of $k$, which is trivial. This shows the first assertion, and the second follows immediately.

Finally we adapt the method of [Tat84, $\S$ II.2 and Theorem IV.5.4] to show the following. Let $n$ denote the number of places of $S$ which split in $K / k$.

LEMMA 6.8.

$$
\left(U_{K, S, T}: U_{k, S, T} U_{K, S, T}^{-}\right)=\frac{2^{n} \#\left(U_{K, S, T}^{-} \cap\{ \pm 1\}\right)}{\# H^{1}\left(\langle\tau\rangle, U_{K, S, T}\right)} .
$$

Proof. The sequence

$$
0 \longrightarrow U_{k, S, T} U_{K, S, T}^{-} \longrightarrow U_{K, S, T} \stackrel{1-\tau}{\longrightarrow} \frac{U_{K, S, T}^{1-\tau}}{\left(U_{K, S, T}^{-}\right)^{2}} \longrightarrow 0
$$

is exact. This shows that $\left(U_{K, S, T}: U_{k, S, T} U_{K, S, T}^{-}\right)=\left(U_{K, S, T}^{1-\tau}:\left(U_{K, S, T}^{-}\right)^{2}\right)$. We also have

$$
\left(U_{K, S, T}^{-}:\left(U_{K, S, T}^{-}\right)^{2}\right)=\left(U_{K, S, T}^{-}: U_{K, S, T}^{1-\tau}\right)\left(U_{K, S, T}^{1-\tau}:\left(U_{K, S, T}^{-}\right)^{2}\right) .
$$

The first factor on the right is $\# H^{1}\left(\langle\tau\rangle, U_{K, S, T}\right)$. The factor on the left can be calculated from the standard decomposition of the finitely generated abelian group $U_{K, S, T}^{-}$. The free rank of this group is $n$, and the cokernel of squaring on the torsion part has the same size as the kernel, which is $U_{K, S, T}^{-} \cap\{ \pm 1\}$. The second factor on the right is what we want to calculate. Hence

$$
2^{n} \#\left(U_{K, S, T}^{-} \cap\{ \pm 1\}\right)=\# H^{1}\left(\langle\tau\rangle, U_{K, S, T}\right)\left(U_{K, S, T}: U_{k, S, T} U_{K, S, T}^{-}\right),
$$

as required.

\subsection{Darmon's conjecture}

We return to the situation of Hypothesis 6.1. We first state Darmon's conjecture in our notation.

Write $\Gamma:=\operatorname{Gal}\left(K\left(\zeta_{S}\right) / K\right)$. For each prime $l_{i} \mid S$ such that $\omega\left(l_{i}\right)=1, l_{i}$ splits into two distinct places $\lambda_{i}$ and $\bar{\lambda}_{i}$ in $K$. Darmon claims that $U_{K, S, \emptyset}^{-}$is a free $\mathbb{Z}$-module [Dar95, $\S 4$ ], but in fact this is not the case since it contains -1 , so actually $U_{K, S, \emptyset}^{-} \cong \mathbb{Z} / 2 \mathbb{Z} \times \mathbb{Z} \# S_{\mathrm{s}}+1$. Taking either $T=\emptyset$ or $T$ such that $U_{K, S, T}$ is torsion-free, choose a basis $u_{1}, \ldots, u_{\# S_{\mathrm{s}}+1}$ for a maximal free subgroup of $U_{K, S, T}^{-}$, which will have index 2 if $T=\emptyset$. Following Darmon, we define a regulator

$$
R_{S, T}:=\sum_{i=1}^{\# S_{\mathrm{s}}+1}(-1)^{i+1} u_{i} \otimes \operatorname{det}\left(f_{\lambda_{k}}\left(u_{j}\right)-1\right)_{k, j} \in U_{K, S, T} \otimes \frac{I_{\Gamma}^{\# S_{\mathrm{s}}}}{I_{\Gamma}^{\# S_{\mathrm{s}}+1}},
$$

where, in the matrix, $k$ runs from 1 to $\# S_{\mathrm{s}}$ and $j$ runs from 1 to $\# S_{\mathrm{s}}+1$, omitting $i$. Note that $R_{S, \emptyset}$ might depend upon the choice of maximal free subgroup if the torsion element -1 is not in the kernel of the local Artin maps.

We state Darmon's conjecture [Dar95, Conjecture 4.3], under the ring automorphism involution of $\mathbb{Z}[\Gamma]$ given by $g \mapsto g^{-1}$, which amounts to a sign change in the statement, and then ignoring all issues of sign. 


\section{A. HAYWARD}

ConjeCture 6.9 (Darmon). We have the following equality in $U_{K\left(\zeta_{S}\right), S} \otimes I_{\Gamma}^{\# S_{\mathrm{s}}} / I_{\Gamma}^{\# S_{\mathrm{s}}+1}$ :

$$
\sum_{\sigma \in \Gamma} \sigma^{-1} \alpha_{S} \otimes \sigma= \pm 2^{\# S_{\mathrm{i}}+1} h_{K, S} R_{S, \emptyset} .
$$

We consider a $T$-modified version. This will fit with our general framework, and avoids the problem of torsion in the unit group. We assume Hypothesis 6.4 part ii which implies that each $v$ in $T$ splits into $w$ and $\bar{w}$ in $K$, with $N_{w}=N_{v}$. Then by Equation (3), we have the following:

$$
h_{\mathbb{Q}, S, T}=h_{\mathbb{Q}, S} \frac{\prod_{v \in T}\left(N_{v}-1\right)}{\left(U_{\mathbb{Q}, S}: U_{\mathbb{Q}, S, T}\right)}, \quad h_{K, S, T}=h_{K, S} \frac{\prod_{v \in T}\left(N_{v}-1\right)^{2}}{\left(U_{K, S}: U_{K, S, T}\right)},
$$

where we note $h_{\mathbb{Q}, S}=1$. The quotient is

$$
\frac{h_{K, S, T}}{h_{\mathbb{Q}, S, T}}=h_{K, S} \frac{\prod_{v \in T}\left(N_{v}-1\right)}{\left(U_{K, S}^{1-\tau}: U_{K, S, T}^{1-\tau}\right)}=h_{K, S} \frac{\prod_{v \in T}\left(N_{v}-1\right)}{\left(U_{K, S}^{-}: U_{K, S, T}^{-}\right) \# H^{1}\left(\langle\tau\rangle, U_{K, S, T}\right)},
$$

using Lemmas 6.6 and 6.7 part ii.

LemMa 6.10. Under Hypothesis 6.4,

$$
\frac{h_{K, S, T}}{h_{\mathbb{Q}, S, T}} \# H^{1}\left(\langle\tau\rangle, U_{K, S, T}\right)
$$

is an integer.

Proof. For $T$ empty, this is clear. Now let $T=\left\{v_{1}, \ldots, v_{n}\right\}$ and choose a place $w_{i}$ of $K$ above each $v_{i}$. Let $K\left(w_{i}\right)$ be the residue field of $K$ at $w_{i}$. Then the natural sequence

$$
0 \longrightarrow U_{K, S, T}^{-} \longrightarrow U_{K, S}^{-} \longrightarrow \bigoplus_{i=1}^{n} K\left(w_{i}\right)^{\times}
$$

is exact. For if $u \in U_{K, S}^{-}$reduces to 1 modulo each $w_{i}$, then $\overline{w_{i}}(u-1)=w_{i}(\bar{u}-1)=w_{i}((1-u) / u)=$ $w_{i}(1-u)>0$, as in Proposition 6.5. Hence $u \in U_{K, S, T} \cap U_{K, S}^{-}=U_{K, S, T}^{-}$.

This shows that $\left(U_{K, S}^{-}: U_{K, S, T}^{-}\right) \mid \prod_{v \in T}\left(N_{v}-1\right)$, and by Equation (18) this gives the result.

We propose the following slight modification of Darmon's conjecture.

Conjecture 6.11. Assume $T$ satisfies Hypothesis 6.4 part ii. Then we have the following equality in $U_{K\left(\zeta_{S}\right), S, T} \otimes I_{\Gamma}^{\# S_{\mathrm{s}}} / I_{\Gamma}^{\# S_{\mathrm{s}}+1}$ :

$$
\sum_{\sigma \in \Gamma} \sigma^{-1} \alpha_{S}^{\delta_{T}^{\omega}} \otimes \sigma= \pm 2^{\# S_{\mathrm{i}}} \frac{h_{K, S, T}}{h_{\mathbb{Q}, S, T}} \# H^{1}\left(\langle\tau\rangle, U_{K, S, T}\right) \#\left(U_{K, S, T}\right)_{\text {tors }} R_{S, T} .
$$

If we put $T=\emptyset$ in this statement, then $h_{\mathbb{Q}, S, T}=1, \# H^{1}\left(\langle\tau\rangle, U_{K, S, T}\right)=1$ (by Lemma 6.7 part i), and $\#\left(U_{K, S, T}\right)_{\text {tors }}=2$. Hence we recover Conjecture 6.9. Next we look at how Conjecture 6.11 varies when we replace $T$ by $T \cup\{v\}$. If $T$ is empty, then for the comparison statement we will have to assume that the regulator in Conjecture 6.9 is calculated with respect to a maximal free subgroup of $U_{K, S}$ which contains $U_{K, S,\{v\}}$. Examining how the various factors change on increase of $T$ shows that Conjecture 6.11 behaves well, and that it follows from Conjecture 6.9 when $U_{K, S, T}^{-}$ can be embedded in a maximal free submodule of $U_{K, S}^{-}$.

The consequence of Darmon's conjecture that we wish to use is the following.

Proposition 6.12. Assume the set-up of Hypothesis 6.1 and let $T$ satisfy Hypothesis 6.4 part ii with $U_{L, S, T}$ torsion-free. Then Conjecture 6.11 implies that for each $\phi \in \operatorname{Hom}_{G}\left(U_{L, S, T}, \mathbb{Z}[G]\right)$, we have

$$
\phi\left(\eta_{\omega}\right) \equiv \pm 2^{\# S_{\mathrm{i}}} \frac{h_{K, S, T}}{h_{\mathbb{Q}, S, T}} \# H^{1}\left(\langle\tau\rangle, U_{K, S, T}\right) \phi^{1}\left(R_{S, T}\right) \quad\left(\bmod I_{G}^{\# S_{\mathrm{s}}+1}\right) .
$$


Proof. Denote as usual $G=\operatorname{Gal}(L / K)$. We apply the natural projection $U_{K\left(\zeta_{S}\right), S} \otimes \mathbb{Z}[\Gamma] \longrightarrow$ $U_{K\left(\zeta_{S}\right), S} \otimes \mathbb{Z}[G]$, which maps the left-hand side of Equation (19) to $\sum_{\sigma \in G} \sigma^{-1}\left(\delta_{T}^{\omega} N_{K\left(\zeta_{S}\right) / L} \alpha_{S}\right) \otimes \sigma$ in $U_{L, S} \otimes \mathbb{Z}[G]$. Then Conjecture 6.11 implies the following equality in $U_{L, S} \otimes I_{G}^{\# S_{\mathrm{s}}} / I_{G}^{\# S_{\mathrm{s}}+1}$ :

$$
\sum_{\sigma \in G} \sigma^{-1} \eta_{\omega} \otimes \sigma= \pm 2^{\# S_{\mathrm{i}}} \frac{h_{K, S, T}}{h_{\mathbb{Q}, S, T}} \# H^{1}\left(\langle\tau\rangle, U_{K, S, T}\right) R_{S, T}
$$

with $\eta_{\omega}$ from Definition 6.3.

Recall the isomorphism (5). Applying the homomorphism

$$
\phi^{1} \otimes \mathrm{id}: U_{L, S, T} \otimes I_{G}^{\# S_{\mathrm{s}}} / I_{G}^{\# S_{\mathrm{s}}+1} \longrightarrow I_{G}^{\# S_{\mathrm{s}}} / I_{G}^{\# S_{\mathrm{s}}+1}
$$

gives the stated result.

\subsection{Factorization of the regulator}

We assume $T$ is such that $U_{L, S, T}$ is torsion-free and that Hypothesis 6.4 is satisfied. We let $u_{1}, \ldots, u_{\# S_{\mathrm{s}}+1}$ be a basis for $U_{K, S, T}^{-}$and $u_{\# S_{\mathrm{s}}+2}, \ldots, u_{2+d^{\prime}}$ be a basis for $U_{\mathbb{Q}, S, T}$. Then these $u_{i}$ form a basis for $U_{\mathbb{Q}, S, T} U_{K, S, T}^{-}$. The index of this group in $U_{K, S, T}$ was calculated in Lemma 6.8. We calculate the regulator from Conjecture 2.6 for these $u_{i}$. Let $\Phi=\phi_{1} \wedge \phi_{2} \in \bigwedge_{G}^{2} \operatorname{Hom}_{\mathbb{Z}[G]}\left(U_{L, S, T}, \mathbb{Z}[G]\right)$. Let $R_{S, T}$ be the regulator defined in $\S 6.5$ in terms of the $u_{i}$. Write $\mathbf{u}_{\mathbb{Q}}=u_{\# S_{\mathrm{s}}+2} \wedge \cdots \wedge u_{2+d^{\prime}}$.

Recall that for each prime $l_{i} \mid S$ such that $\omega\left(l_{i}\right)=1, l_{i}$ splits into distinct places $\lambda_{i}, \bar{\lambda}_{i}$ in $K$. The other $\# S_{\mathrm{i}}$ primes dividing $S$ are inert in $K / \mathbb{Q}$, and will be denoted $q_{1}, \ldots, q_{\# S_{\mathrm{i}}}$. For reference, we summarize $S_{\mathbb{Q}}$ and $S_{K}$ :

$$
\begin{gathered}
S_{\mathbb{Q}}=\left\{\infty, l_{1}, \ldots, l_{\# S_{\mathrm{s}}}, q_{1}, \ldots, q_{\# S_{\mathrm{i}}}\right\}, \\
S_{1, \mathbb{Q}}=\{\infty\}, \quad r=1, \quad \# S_{\mathbb{Q}}=r+d+1, \quad \text { so } d=\# S_{\mathrm{s}}+\# S_{\mathrm{i}} ; \\
S_{K}=\left\{\infty_{L}, \overline{\infty_{L}}, \lambda_{1}, \ldots, \lambda_{\# S_{\mathrm{s}}}, \bar{\lambda}_{1}, \ldots, \bar{\lambda}_{\# S_{\mathrm{s}}}, q_{1}, \ldots, q_{\# S_{\mathrm{i}}}\right\}, \\
S_{1, K}=\left\{\infty_{L}, \bar{\infty}_{L}\right\}, \quad r^{\prime}=2, \quad \# S_{K}=r^{\prime}+d^{\prime}+1, \quad \text { so } d^{\prime}=2 \# S_{\mathrm{s}}+\# S_{\mathrm{i}} .
\end{gathered}
$$

Proposition 6.13. We have the following equality in $\mathbb{Z}[G] / I_{G}^{d^{\prime}+1}$ :

$$
\operatorname{Reg}_{L / K}\left(\tilde{\Phi}\left(u_{1} \wedge \cdots \wedge u_{2+d^{\prime}}\right)\right)= \pm 2^{\# S_{\mathrm{s}}}\left|\begin{array}{cc}
2^{\# S_{\mathrm{i}}-1} \phi_{1}^{1}\left(R_{S, T}\right) & 2^{\# S_{\mathrm{i}}-1} \phi_{2}^{1}\left(R_{S, T}\right) \\
\operatorname{Reg}_{\tilde{L} / \mathbb{Q}}\left(\tilde{\phi}_{1}\left(\mathbf{u}_{\mathbb{Q}}\right)\right) & \operatorname{Reg}_{\tilde{L} / \mathbb{Q}}\left(\tilde{\phi}_{2}\left(\mathbf{u}_{\mathbb{Q}}\right)\right)
\end{array}\right| .
$$

Proof. We saw in Lemma 2.4 that

$$
\tilde{\Phi}\left(u_{1} \wedge \cdots \wedge u_{2+d^{\prime}}\right)=\sum_{\sigma \in\left[\begin{array}{c}
2+d^{\prime} \\
2
\end{array}\right]} \operatorname{sign}(\sigma)\left|\begin{array}{ll}
\phi_{1}^{1}\left(u_{\sigma(1)}\right) & \phi_{2}^{1}\left(u_{\sigma(1)}\right) \\
\phi_{1}^{1}\left(u_{\sigma(2)}\right) & \phi_{2}^{1}\left(u_{\sigma(2)}\right)
\end{array}\right| u_{\sigma(3)} \wedge \cdots \wedge u_{\sigma\left(2+d^{\prime}\right)} .
$$

The terms $u_{\sigma(3)} \wedge \cdots \wedge u_{\sigma\left(2+d^{\prime}\right)}$ are made by choosing two of the $u_{i}$ for the integer determinant. So each $\sigma$ excludes 0,1 or 2 units of the $U_{K, S, T}^{-}$basis from the wedge of units. Let $m_{\sigma}=\#\left(\sigma\left(\left\{3,4, \ldots, 2+d^{\prime}\right\}\right)\right.$ $\left.\cap\left\{1, \ldots, \# S_{\mathrm{s}}+1\right\}\right)$ be the number of minus-units included in $u_{\sigma(3)} \wedge \cdots \wedge u_{\sigma\left(2+d^{\prime}\right)}$ in the term corresponding to $\sigma$, so $m_{\sigma}=\# S_{\mathrm{s}}-1, \# S_{\mathrm{s}}$ or $\# S_{\mathrm{s}}+1$.

We calculate our matrix with respect to the following places of $K$, using Hypothesis 6.4 to exclude $q_{\# S_{\mathrm{i}}}$ :

$$
\lambda_{1}, \ldots, \lambda_{\# S_{\mathrm{s}}}, \bar{\lambda}_{1}, \ldots, \bar{\lambda}_{\# S_{\mathrm{s}}}, q_{1}, \ldots, q_{\# S_{\mathrm{i}}-1}
$$




\section{A. HAYWARD}

This means we have the determinant of the following $d^{\prime} \times d^{\prime}$ matrix to calculate for $\operatorname{Reg}_{L / K}\left(u_{\sigma(3)} \wedge\right.$ $\left.\cdots \wedge u_{\sigma\left(2+d^{\prime}\right)}\right)$ :

\begin{tabular}{|c|c|c|c|}
\hline & $\lambda_{1}, \ldots, \lambda_{\# S_{\mathrm{s}}}$ & $\bar{\lambda}_{1}, \ldots, \bar{\lambda}_{\# S_{\mathrm{s}}}$ & $q_{1}, \ldots, q_{\# S_{\mathrm{i}}-1}$ \\
\hline $\begin{array}{c}u_{\sigma(3)} \\
\vdots \\
u_{\sigma\left(m_{\sigma}+2\right)}\end{array}$ & $f_{\lambda_{j}}\left(u_{i}\right)-1$ & $f_{\overline{\lambda_{j}}}\left(u_{i}\right)-1$ & $f_{q_{j}}\left(u_{i}\right)-1$ \\
\hline $\begin{array}{c}u_{\sigma\left(m_{\sigma}+3\right)} \\
\vdots \\
u_{\sigma\left(2+d^{\prime}\right)}\end{array}$ & $f_{\lambda_{j}}\left(u_{i}\right)-1$ & $f_{\overline{\lambda_{j}}}\left(u_{i}\right)-1$ & $f_{q_{j}}\left(u_{i}\right)-1$ \\
\hline
\end{tabular}

where the units at the top are in $U_{K, S, T}^{-}$and the units at the bottom are in $U_{\mathbb{Q}, S, T}$. We will distinguish between the cases where $m_{\sigma}$ takes the different values.

First consider the case $m_{\sigma}=\# S_{\mathrm{s}}+1$. We may add the column for $\lambda_{j}$ to the column for $\overline{\lambda_{j}}$ for $j=1, \ldots, \# S_{\mathrm{s}}$ without altering the value of the determinant. The $(i, j)$ th entry in the top-centre $\left(\# S_{\mathrm{s}}+1\right) \times\left(\# S_{\mathrm{s}}\right)$ block is then congruent $\bmod I_{G}^{2}$ to $f_{\lambda_{j}}\left(\bar{u}_{i} u_{i}\right)-1=0$. Next we note that, for each $q_{j}$, the local extension $K_{q_{j}} / \mathbb{Q}_{q_{j}}$ has degree two, and $f_{q_{j}}\left(u_{i}\right)$ only depends on the norm of $u_{i}$ in this local extension. If $u_{i} \in U_{K, S, T}^{-}$then $u_{i}^{1+\tau}=1$. Hence $f_{q_{j}}\left(u_{i}\right)-1=0$ for these $u_{i}$. Therefore the entire top-right $\left(\# S_{\mathrm{s}}+1\right) \times\left(\# S_{\mathrm{s}}+\# S_{\mathrm{i}}-1\right)$ block is zero. Hence there are at most \#S columns which are non-zero in their first $\# S+1$ rows. Therefore the determinant is zero.

Now in Equation (21) we subtract the column for $\bar{\lambda}_{j}$ from the column for $\lambda_{j}$ for $j=1, \ldots, \# S_{\mathrm{s}}$ to show that the determinant is the same as the determinant of the following matrix:

\begin{tabular}{|c|c|c|c|}
\hline & $\lambda_{1}, \ldots, \lambda_{\# S_{\mathrm{s}}}$ & $\bar{\lambda}_{1}, \ldots, \bar{\lambda}_{\# S_{\mathrm{s}}}$ & $q_{1}, \ldots, q_{\# S_{\mathrm{i}}-1}$ \\
\hline $\begin{array}{c}u_{\sigma(3)} \\
\vdots \\
u_{\sigma\left(m_{\sigma}+2\right)}\end{array}$ & $f_{\lambda_{j}}\left(u_{i}\right)-f_{\overline{\lambda_{j}}}\left(u_{i}\right)$ & $f_{\lambda_{j}}\left(u_{i}\right)-1$ & $f_{q_{j}}\left(u_{i}\right)-1$ \\
\hline $\begin{array}{c}u_{\sigma\left(m_{\sigma}+3\right)} \\
\vdots \\
u_{\sigma\left(2+d^{\prime}\right)}\end{array}$ & 0 & $f_{\overline{\lambda_{j}}}\left(u_{i}\right)-1$ & $f_{q_{j}}\left(u_{i}\right)-1$ \\
\hline
\end{tabular}

If $m_{\sigma}=\# S_{\mathrm{s}}-1$, the first $\# S_{\mathrm{s}}$ columns have all zeros except perhaps in the first $\# S_{\mathrm{s}}-1$ rows. Therefore the determinant is again zero.

We are left with the case $m_{\sigma}=\# S_{\mathrm{s}}$. In this case matrix (22) is block-upper-triangular. Let us consider the top-left $\left(\# S_{\mathrm{s}}\right) \times\left(\# S_{\mathrm{s}}\right)$ block first. We note that $f_{\lambda_{j}}\left(u_{\sigma(i)}\right)-f_{\overline{\lambda_{j}}}\left(u_{\sigma(i)}\right) \equiv 2\left(f_{\lambda_{j}}\left(u_{\sigma(i)}\right)-1\right)$ $\left(\bmod I_{G}^{2}\right)$. So the top-left block has determinant $2^{\# S_{\mathrm{s}}} \operatorname{det}\left(f_{\lambda_{j}}\left(u_{\sigma(i)}\right)-1\right)_{i, j}$. Note the relationship to the regulator $R_{S, T}$ of (17).

Now we calculate the determinant of the bottom-right block. We have $f_{\overline{\lambda_{j}}}(u)=f_{l_{j}}^{\prime}(u), f_{q_{j}}(u)=$ $f_{q_{j}}^{\prime}(u)^{2}$ for each $j$ and each $u$ appearing, where the $f^{\prime}$ denote the local symbols coming from the extension $\tilde{L} / \mathbb{Q}$. So the bottom-right block is $2^{\# S_{\mathrm{i}}-1} \operatorname{Reg}_{\tilde{L} / \mathbb{Q}}\left(u_{\sigma\left(m_{\sigma}+3\right)} \wedge \cdots \wedge u_{\sigma\left(2+d^{\prime}\right)}\right)$.

Referring back to Equation (20), the only terms which appear in the sum after applying $\operatorname{Reg}_{L / K}$ are those for $\sigma$ such that $\sigma(1) \leqslant \# S_{\mathrm{s}}+1$ and $\sigma(2)>\# S_{\mathrm{s}}+1$. We put this in correspondence with a pair $(i, j), 1 \leqslant i \leqslant \# S_{\mathrm{s}}+1,1 \leqslant j \leqslant d+1$ such that $\sigma(1)=i, \sigma(2)=\# S_{\mathrm{s}}+1+j$. Then one may check that $\operatorname{sign}(\sigma)=(-1)^{\# S_{\mathrm{s}}}(-1)^{i+1}(-1)^{j+1}$.

Putting all this together with Equation (20) gives the stated result, with sign $(-1)^{\# S_{\mathrm{s}}}$ on the right. 


\section{ABELIAN $L$-FUNCTIONS}

\subsection{Base change for the congruence}

We are now ready to show the base change statement for Conjecture 2.6.

TheOREm 6.14. We use the set-up of Hypothesis 6.1, assume Hypothesis 6.4 and use the definition of $u_{i}$ from $\S 6.6$.

Assume Conjecture 2.6 holds for the extension $\tilde{L} / \mathbb{Q}$, i.e. that

$$
\phi\left(\eta_{\tilde{L} / \mathbb{Q}}\right) \equiv \pm h_{\mathbb{Q}, S, T} \operatorname{Reg}_{\tilde{L} / K}\left(\tilde{\phi}\left(u_{\# S_{\mathrm{s}}+2} \wedge \cdots \wedge u_{2+d^{\prime}}\right)\right) \quad\left(\bmod I_{G}^{d+1}\right)
$$

for all $\phi \in \operatorname{Hom}_{G}\left(U_{\tilde{L}, S, T}, \mathbb{Z}[G]\right)$. Assume also that the modified Darmon Conjecture 6.11 holds. Then Conjecture 2.6 holds for the extension $L / K$ up to a power of 2. Explicitly, for all $\Phi \in$ $\bigwedge_{G}^{2} \operatorname{Hom}_{G}\left(U_{L, S, T}, \mathbb{Z}[G]\right)$, we have

$$
4 \cdot 2^{\# S_{\mathrm{s}}} \Phi\left(\eta_{L / K}\right) \equiv \pm 4 \cdot 2^{\# S_{\mathrm{s}}} h_{K, S, T} \operatorname{Reg}_{L / K}\left(\tilde{\Phi}\left(\epsilon_{1} \wedge \cdots \wedge \epsilon_{2+d^{\prime}}\right)\right) \quad\left(\bmod I_{G}^{d^{\prime}+1}\right),
$$

where the $\epsilon_{i}$ form a $\mathbb{Z}$-basis for $U_{K, S, T}$.

Proof. Write $\mathbf{u}=u_{1} \wedge \cdots \wedge u_{2+d^{\prime}}, \epsilon=\epsilon_{1} \wedge \cdots \wedge \epsilon_{2+d^{\prime}}$. Set $\Phi=\phi_{1} \wedge \phi_{2}$, and recall from $\S 6.3$ that $4 \eta_{L / K}=\eta_{\omega} \wedge \eta_{\tilde{L} / \mathbb{Q}}$. The conjectures tell us, using Proposition 6.12, that

$$
4 \Phi\left(\eta_{L / K}\right)=\left|\begin{array}{cc}
\phi_{1}\left(\eta_{\omega}\right) & \phi_{2}\left(\eta_{\omega}\right) \\
\phi_{1}\left(\eta_{\tilde{L} / \mathbb{Q}}\right) & \phi_{2}\left(\eta_{\tilde{L} / \mathbb{Q}}\right)
\end{array}\right| \equiv \pm\left|\begin{array}{cc}
2^{\# S_{\mathrm{i}}} \frac{h_{K, S, T}}{h_{\mathbb{Q}, S, T}} \# H^{1} \phi_{1}^{1}\left(R_{S, T}\right) & 2^{\# S_{\mathrm{i}}} \frac{h_{K, S, T}}{h_{\mathbb{Q}, S, T}} \# H^{1} \phi_{2}^{1}\left(R_{S, T}\right) \\
h_{\mathbb{Q}, S, T} \operatorname{Reg}_{\tilde{L} / \mathbb{Q}}\left(\tilde{\phi}_{1}\left(\mathbf{u}_{\mathbb{Q}}\right)\right) & h_{\mathbb{Q}, S, T} \operatorname{Reg}_{\tilde{L} / \mathbb{Q}}\left(\tilde{\phi}_{2}\left(\mathbf{u}_{\mathbb{Q}}\right)\right)
\end{array}\right|
$$

modulo $I_{G}^{d+1+\# S_{\mathrm{s}}+1}$, where $\# H^{1}=\# H^{1}\left(\langle\tau\rangle, U_{K, S, T}\right)$ and $R_{S, T}$ is calculated with respect to the $\mathbb{Z}$-basis $u_{1}, \ldots, u_{\# S_{\mathrm{s}}+1}$ of $U_{K, S, T}^{-}$. Noting that $d^{\prime}=d+\# S_{\mathrm{s}}$, this is

$$
\equiv \pm 2^{\# S_{\mathrm{i}}} h_{K, S, T} \# H^{1}\left|\begin{array}{cc}
\phi_{1}^{1}\left(R_{S, T}\right) & \phi_{2}^{1}\left(R_{S, T}\right) \\
\operatorname{Reg}_{\tilde{L} / \mathbb{Q}}\left(\tilde{\phi}_{1}\left(\mathbf{u}_{\mathbb{Q}}\right)\right) & \operatorname{Reg}_{\tilde{L} / \mathbb{Q}}\left(\tilde{\phi}_{2}\left(\mathbf{u}_{\mathbb{Q}}\right)\right)
\end{array}\right| \quad\left(\bmod I_{G}^{d^{\prime}+2}\right) .
$$

Hence by Proposition 6.13 , we have $4 \cdot 2^{\# S_{\mathrm{s}}} \Phi\left(\eta_{L / K}\right) \equiv \pm h_{K, S, T} \cdot 2 \# H^{1} \operatorname{Reg}(\tilde{\Phi}(\mathbf{u}))\left(\bmod I_{G}^{d^{\prime}+1}\right)$. Now we know from Lemma 6.8 that $\left(U_{K, S, T}: U_{\mathbb{Q}, S, T} U_{K, S, T}^{-}\right)=2^{\# S_{\mathrm{s}}+1} / \# H^{1}$, so $\mathbf{u}=\left(2^{\# S_{\mathrm{s}}+1} / \# H^{1}\right) \epsilon$. This gives the result.

Note that if $\# G$ is odd, this last congruence is the full statement of Conjecture 2.6.

Remark 6.15. Using the method of [Dar95, Lemma 8.1], it is possible to prove that $\phi\left(N_{K\left(\zeta_{S}\right) / L} \alpha_{S}\right) \in$ $I_{G}^{\# S_{\mathrm{s}}}$ for all $\phi \in \operatorname{Hom}_{G}\left(U_{L, S}^{-}, \mathbb{Z}[G]\right)$, without assuming the validity of Darmon's conjecture. It then follows that $\phi^{\prime}\left(\eta_{\omega}\right) \in I_{G}^{\# S_{\mathrm{s}}}$ for all $\phi^{\prime} \in \operatorname{Hom}_{G}\left(U_{L, S, T}, \mathbb{Z}[G]\right)$. Thus if Conjecture 2.6 holds for $\tilde{L} / \mathbb{Q}$, then for $L / K$ we have $4 \Phi\left(\eta_{L / K}\right) \in I_{G}^{d^{\prime}}$, for all $\Phi \in \bigwedge_{G}^{2} \operatorname{Hom}_{G}\left(U_{L, S, T}, \mathbb{Z}[G]\right)$, consistent with the 'order of vanishing' implied by Conjecture 2.6.

\section{Base change via Gross's conjecture on the $L$-functions of tori}

In $\S 8$ of [Gro88], Gross makes a conjecture motivated by considering algebraic tori. Similarly to Darmon's later conjecture, this involves a quadratic extension of the base field and consideration of the 'minus-units' in this extension. It also involves a ' $\Theta$ ' element which is twisted by the non-trivial character of the extension. In the previous section we saw that Darmon's conjecture, which was related to the first derivative of the relative factor in Equation (14), gave us a base change property for Conjecture 2.6 where the order of vanishing, $r$, increased by 1. Gross's conjecture, by contrast, concerns the value (zeroth derivative) of the relative factor and correspondingly it gives us a base change property where $r$ does not change. 


\section{A. HAYWARD}

\subsection{Set-up and calculation of $\eta$}

Our set-up is as follows. Let $k$ be a global field and $\tilde{L} / k$ and $K / k$ linearly disjoint abelian extensions, with $[K: k]=2$. Let $\omega: \operatorname{Gal}(K / k) \longrightarrow\{ \pm 1\}$ be the non-trivial character of $K / k$. Setting $L=\tilde{L} K$, we are in the situation of $\S 6.1$. Write $G=\operatorname{Gal}(L / K)=\operatorname{Gal}(\tilde{L} / k)$. Let $S=S_{k}$ be a set of places of $k$ containing all infinite places and all places ramifying in $L / k$. That is, both $\tilde{L} / k$ and $K / k$ are unramified outside $S$. Take $T=T_{k}$ such that $U_{L, S, T}$ is torsion-free. We define $n$ to be the number of places in $S$ splitting in the quadratic extension $K / k$, and refer to the other $\# S-n$ inert or ramified places as non-split. We write $\tau$ for the non-trivial automorphism of this extension. This is the situation of $\S 8$ of [Gro88] except we have an unfortunate clash of notation, summarized in the following table:

\begin{tabular}{c||c|c|c|c} 
Gross's notation & $L$ & $K$ & $\chi$ & $\sigma$ \\
\hline Our notation & $K$ & $\tilde{L}$ & $\omega$ & $\tau$
\end{tabular}

Assume there are $r$ places $S_{1, k}$ in $S_{k}$ splitting completely in $\tilde{L} / k$. Then all the places above these in $K$ split completely in $L / K$, and there are at least $r$ of them, so the two sets of data $L / K, S_{K} \supseteq S_{1, K}, T_{K}, r$ and $\tilde{L} / k, S_{k} \supseteq S_{1, k}, T_{k}, r$ both satisfy Hypothesis 2.1. Differentiating the base-change factorization of the $L$-functions (13) $r$ times and evaluating at $s=0$ gives

$$
\Theta_{L / K, S_{K}, T_{K}}^{r}(0)=\Theta_{L / K / k, S_{k}, T_{k}}(0, \omega) \Theta_{\tilde{L} / k, S_{k}, T_{k}}^{r}(0) .
$$

The base-change factor $\Theta_{L / K / k, S_{k}, T_{k}}(0, \omega)$ lies in $\mathbb{Z}[G]$ by the argument following [Gro88, Equation (8.7)], where the corresponding element is denoted $\theta_{G}(\chi)$. Gross's tori conjecture concerns this element, and we will show that its validity would imply that Conjecture 2.6 for $L / K, S_{K} \supseteq$ $S_{1, K}, T_{K}, r$ (weakened by powers of 2 , similarly to the case of Darmon's conjecture) follows from the conjecture for $\tilde{L} / k, S_{k} \supseteq S_{1, k}, T_{k}, r$.

First note that we may assume that the $r$ places in $S_{1, k}$ are non-split in $K / k$, since otherwise more than $r$ places in $S_{K}$ split in $L / K$ and Conjecture 2.6 already holds for $L / K, S_{K}, T_{K}, r$ by Proposition 3.10. We also impose the following assumption, which is the same as Hypothesis 6.4 part i.

Hypothesis 7.1. There is a place in $S_{k}-S_{1, k}$ which is non-split in $K / k$. That is, $d \geqslant n$.

Let $v_{0}$ be such a place. Write $S_{1, k}=\left\{v_{1}, \ldots, v_{r}\right\}$. Choose $w_{i}$ a place of $L$ above $v_{i}$ for $i=$ $0,1, \ldots, r$. Set $\mathbf{b}_{L}=\left(w_{1}-w_{0}\right) \wedge \cdots \wedge\left(w_{r}-w_{0}\right)$. Write $\tilde{w}_{i}$ for the place of $\tilde{L}$ induced by $w_{i}$ for $i=0, \ldots, r$, and set $\mathbf{b}_{\tilde{L}}=\left(\tilde{w}_{1}-\tilde{w}_{0}\right) \wedge \cdots \wedge\left(\tilde{w}_{r}-\tilde{w}_{0}\right)$. Then with these choices of the $W$ in the definition of $\eta$, we have that $\eta_{\tilde{L} / k} \in \mathbb{C} \bigwedge_{\mathbb{Z}[G]}^{r} U_{\tilde{L}, S, T}$ is defined by the equation

$$
\lambda_{\tilde{L}}\left(\eta_{\tilde{L} / k}\right)=\Theta_{\tilde{L} / k, S_{k}, T_{k}}^{r}(0) \mathbf{b}_{\tilde{L}} .
$$

The commutative diagrams $(16)$ hold here (with $k$ instead of $\mathbb{Q}$ ). Since $v_{0}, \ldots, v_{r}$ are all non-split in $K / k$, there is a unique $w_{i}$ over $\tilde{w}_{i}$ for $i=0, \ldots, r$. Hence

$$
\lambda_{L}\left(\eta_{\tilde{L} / k}\right)=\Theta_{\tilde{L} / k, S_{k}, T_{k}}^{r}(0)\left(\left(2 w_{1}-2 w_{0}\right) \wedge \cdots \wedge\left(2 w_{r}-2 w_{0}\right)\right)=2^{r} \Theta_{\tilde{L} / k, S_{k}, T_{k}}^{r}(0) \mathbf{b}_{L} .
$$

Therefore we have, using Equation $(23), \lambda_{L}\left(\eta_{\tilde{L} / k}^{\Theta_{L / K / k, S, T}(0, \omega)}\right)=2^{r} \Theta_{L / K, S_{K}, T_{K}}^{r}(0) \mathbf{b}_{L}$. It follows that

$$
\eta_{L / K}=\frac{1}{2^{r}} \eta_{\tilde{L} / k}^{\Theta_{L / K / k, S, T}(0, \omega)}
$$




\section{ABELIAN $L$-FUNCTIONS}

\subsection{Regulator calculations}

Keeping the assumptions of $\S 7.1$, we now go on to study the regulators involved in the various conjectures. Break up $S_{k}$ as follows:

$$
S_{k}=\{\overbrace{v_{1}, \ldots, v_{r}}^{S_{1, k}, \text { non-split in } K} \overbrace{v_{r+1}, \ldots, v_{r+n}}^{n \text { split in } K}, \overbrace{v_{r+n+1}, \ldots, v_{r+d+1}}^{d-n+1 \text { non-split in } K}\} .
$$

Note that $d-n+1>0$ by Hypothesis 7.1. Choose $v_{i}^{\prime}$ to be place of $K$ above $v_{i}$ for $i=1, \ldots, r+d+1$. Choose a $\mathbb{Z}$-basis $\mu_{1}, \ldots, \mu_{n}$ of $U_{K, S, T}^{-}$. Then we can define a minus-unit regulator $R_{G}^{-} \in \mathbb{Z}[G] / I_{G}^{n+1}$ by the determinant of the $n \times n$ matrix with $(i, j)$ th entry $f_{v_{r+j}^{\prime}}\left(\mu_{i}\right)-1$ for $1 \leqslant i, j \leqslant n$. This is denoted $\operatorname{det}_{G}\left(\lambda_{\tau}\right)$ in [Gro88].

We choose a $\mathbb{Z}$-basis $u_{1}, \ldots, u_{r+d+n}$ for $U_{K, S, T}$ such that $u_{1+n}, \ldots, u_{r+d+n}$ is a basis for $U_{k, S, T}$, which is possible by Lemma 3.1 .

The analogue of Proposition 6.13 in this situation is the following.

Proposition 7.2. We have the following in $\mathbb{Z}[G] / I_{G}^{d+n+1}$ :

$$
\operatorname{Reg}_{L / K}\left(\tilde{\Phi}\left(u_{1} \wedge \cdots \wedge u_{r+d+n}\right)\right)= \pm 2^{d-n} R_{G}^{-} \operatorname{Reg}_{\tilde{L} / k}\left(\tilde{\Phi}\left(u_{1+n} \wedge \cdots \wedge u_{r+d+n}\right)\right)\left(U_{K, S, T}^{-}: U_{K, S, T}^{1-\tau}\right) .
$$

Proof. The regulator on the left is

$$
\sum_{\sigma \in\left[\begin{array}{c}
r+d+n \\
r
\end{array}\right]} \operatorname{sign}(\sigma) \operatorname{det}\left(\phi_{j}^{1}\left(u_{\sigma(i)}\right)\right)_{1 \leqslant i, j \leqslant r} \operatorname{Reg}_{L / K}\left(u_{\sigma(r+1)} \wedge \cdots \wedge u_{\sigma(r+d+n)}\right),
$$

where, after manipulations as in the proof of Proposition 6.13, $\operatorname{Reg}_{L / K}\left(u_{\sigma(r+1)} \wedge \cdots \wedge u_{\sigma(r+d+n)}\right)$ is seen to be the determinant in $\mathbb{Z}[G] / I_{G}^{d+n+1}$ of the matrix

\begin{tabular}{c||c|c|c} 
& $v_{r+1}^{\prime}, \ldots, v_{r+n}^{\prime}$ & $\bar{v}_{r+1}^{\prime}, \ldots, \bar{v}_{r+n}^{\prime}$ & $v_{r+n+1}^{\prime}, \ldots, v_{r+n+d}^{\prime}$ \\
\hline \hline$u_{\sigma(r+1)}$ & $f_{v_{j}^{\prime}}\left(u_{i}^{1-\tau}\right)-1$ & $f_{v_{j}^{\prime}}\left(u_{i}^{\tau}\right)-1$ & $f_{v_{j}^{\prime}}\left(u_{i}\right)-1$ \\
$\vdots$ \\
$u_{\sigma\left(r+m_{\sigma}\right)}$ & & & \\
\hline$u_{\sigma\left(r+m_{\sigma}+1\right)}$ & & $f_{v_{j}}\left(u_{i}\right)-1$ & $2\left(f_{v_{j}}\left(u_{i}\right)-1\right)$ \\
$\begin{array}{c}u_{\sigma(r+d+n)} \\
u^{\prime}\end{array}$ & 0 & &
\end{tabular}

in which $m_{\sigma}=\# \sigma(\{r+1, \ldots, r+d+n\}) \cap\{1, \ldots, n\}$. Now if $m_{\sigma}<n$ then this determinant is clearly 0. So for non-zero terms in the sum (25) we must have $m_{\sigma}=n$, i.e. $\sigma(r+1)=$ $1, \ldots, \sigma(r+n)=n$. Then $u_{\sigma(r+1)}^{1-\tau}, \ldots, u_{\sigma(r+n)}^{1-\tau}$ is a $\mathbb{Z}$-basis for $U_{K, S, T}^{1-\tau}$ and so the determinant of the top-left $n \times n$ block is $\left(U_{K, S, T}^{-}: U_{K, S, T}^{1-\tau}\right) R_{G}^{-}$. The determinant of the bottom-right $d \times d$ block is $2^{d-n} \operatorname{Reg}_{\tilde{L} / k}\left(u_{\sigma(r+n+1)} \wedge \cdots \wedge u_{\sigma(r+d+n)}\right)$.

Note that for such $\sigma$, the map $\sigma \circ\left(\begin{array}{llll}1 & 2 & \ldots & r+n\end{array}\right)^{r}$ is a permutation of $\{n+1, \ldots, r+d+n\}$ of the form $n+k \mapsto n+\sigma^{\prime}(k)$ for $\sigma^{\prime} \in\left[\begin{array}{c}r+d \\ r\end{array}\right]$. We have

$$
\begin{aligned}
& \operatorname{Reg}_{L / K}\left(\tilde{\Phi}\left(u_{1} \wedge \cdots \wedge u_{r+d+n}\right)\right) \\
& \quad=\left(U_{K, S, T}^{-}: U_{K, S, T}^{1-\tau}\right) R_{G}^{-} \sum_{\sigma^{\prime}}\left((-1)^{r(r+n+1)} \operatorname{sign}\left(\sigma^{\prime}\right) \operatorname{det}\left(\phi_{j}^{1}\left(u_{n+\sigma^{\prime}(i)}\right)\right)_{1 \leqslant i, j \leqslant r}\right. \\
& \left.\quad \times \operatorname{Reg}_{\tilde{L} / k}\left(u_{n+\sigma^{\prime}(r+1)} \wedge \cdots \wedge u_{n+\sigma^{\prime}(r+d)}\right)\right),
\end{aligned}
$$

which gives the result. 


\section{A. HAYWARD}

\subsection{Gross's conjecture on the $L$-functions of tori}

We will now state Conjecture 8.8 of [Gro88]. The analytic class number formula makes it possible to calculate the coefficient of the leading term of the Taylor expansion of $L_{K / k, S, T}(s, \omega)$ at $s=0$, as in [Tat84, ch. II, $\S 2$ ]. It is $m_{\omega} R^{-}$, where $R^{-}$is a logarithmic regulator calculated with respect to bases of the minus-parts of $U_{K, S, T}$ and $X_{S_{K}}$, and

$$
m_{\omega}= \pm \frac{h_{K, S, T}}{h_{k, S, T}} 2^{\# S-n-1}\left(U_{K, S, T}^{-}: U_{K, S, T}^{1-\tau}\right) .
$$

The reader is warned that the factor $2^{\# S-n-1}$ is missing in Equation (8.5) of [Gro88].

Lemma 7.3. Assuming Hypothesis 7.1, $m_{\omega}$ is an integer.

Proof. The hypothesis shows that $\# S-n-1 \geqslant 0$. Also $K / k$ is a quadratic extension unramified outside $S$ such that at least one place in $S$ is inert. Therefore Lemma 4.6 part i shows that $h_{k, S, T}$ divides $h_{K, S, T}$. This gives the result.

We can now state Gross's tori conjecture, which in our set-up, assuming Hypothesis 7.1 in order to have the conclusion of Lemma 7.3, states the following.

Conjecture 7.4 (Gross). We have

$$
\Theta_{L / K / k, S, T}(0, \omega) \equiv m_{\omega} R_{G}^{-} \quad\left(\bmod I_{G}^{n+1}\right)
$$

\subsection{Base change}

TheOREM 7.5. Let $\tilde{L} / k, K / k$ be finite linearly disjoint abelian extensions of a global field $k$, with $[K: k]=2$. Set $L=\tilde{L} K$. Assume $S=S_{k}, T=T_{k}$ are such that $L / k$ is unramified outside $S_{k}$ and $U_{L, S, T}$ is torsion-free. Let $S_{1} \subseteq S$ be a set of $r$ places which split in $\tilde{L} / k$ but not in $K / k$. Assume Hypothesis 7.1 for these data.

Assume that Conjecture 2.6 holds for $\tilde{L} / k, S, T, r$ and that Conjecture 7.4 holds. Then for all $\Phi \in \bigwedge_{\mathbb{Z}[G]}^{r} \operatorname{Hom}_{\mathbb{Z}[G]}\left(U_{L, S, T}, \mathbb{Z}[G]\right)$ we have

$$
2^{r} \Phi\left(\eta_{L / K}\right) \equiv \pm 2^{r} h_{K, S, T} \operatorname{Reg}_{G}^{\Phi} \quad\left(\bmod I_{G}^{d+n+1}\right) .
$$

That is, the conclusion of Conjecture 2.6 for $L / K, S_{K}, T_{K}, r$ holds with a factor of $2^{r}$ on each side.

Proof. By Equation (24) we have $2^{r} \Phi\left(\eta_{L / K}\right)=\Theta_{L / K / k, S, T}(0, \omega) \Phi\left(\eta_{\tilde{L} / k}\right)$. Multiplying the congruences of Conjecture 7.4 and Conjecture 2.6 gives

$$
\begin{aligned}
2^{r} \Phi\left(\eta_{L / K}\right) \equiv & \pm \frac{h_{K, S, T}}{h_{k, S, T}} 2^{r+d-n}\left(U_{K, S, T}^{-}: U_{K, S, T}^{1-\tau}\right) R_{G}^{-} h_{k, S, T} \\
& \times \operatorname{Reg}_{\tilde{L} / k}\left(\tilde{\Phi}\left(u_{1+n} \wedge \cdots \wedge u_{r+d+n}\right)\right) \quad\left(\bmod I_{G}^{d+n+2}\right) \\
\equiv & \pm 2^{r} h_{K, S, T} \operatorname{Reg}_{L / K}\left(\tilde{\Phi}\left(u_{1} \wedge \cdots \wedge u_{r+d+n}\right)\right) \quad\left(\bmod I_{G}^{d+n+1}\right)
\end{aligned}
$$

by the regulator calculation in Proposition 7.2.

Note that if $r=0$ then this shows that, under Hypothesis 7.1, Gross's conjecture on tori actually gives a base-change property with no weakening factor for Conjecture 4.1 in [Gro88].

\section{ACKNOWLEDGEMENTS}

It is a pleasure to thank David Burns for his help and guidance while writing this paper, and Radan Kučera for many useful comments. 


\section{ABELiAn $L$-FUnCtions}

\section{REFERENCES}

Bur01 D. Burns, On refined class number formulas for higher derivatives of L-series, Preprint (2001).

Bur03 D. Burns, On relations between derivatives of abelian L-functions at $s=0$, Manuscript in preparation.

BF01 D. Burns and M. Flach, Tamagawa numbers for motives with (non-commutative) coefficients, Doc. Math. 6 (2001), 501-570.

Dar95 H. Darmon, Thaine's method for circular units and a conjecture of Gross, Can. J. Math. 47 (1995), 302-317.

Gro88 B. H. Gross, On the values of abelian L-functions at $s=0$, J. Faculty Sci. Univ. Tokyo, Sect 1A, Math. 35 (1988), 177-197.

GK02 C. Greither and R. Kučera, The lifted root number conjecture for fields of prime degree over the rationals: an approach via trees and Euler systems, Ann. Inst. Fourier 52(3) (2002), 735-777.

GK03 C. Greither and R. Kučera, Annihilators for the class group of a cyclic field of prime power degree, Acta Arith., to appear.

Pop99 C. D. Popescu, On a refined Stark conjecture for function fields, Compositio Math. 116 (1999), 321-367.

Pop02 C. D. Popescu, Base change for Stark-type conjectures 'Over $\mathbb{Z}$ ', J. Reine Angew. Math. 542 (2002), $85-111$.

Rim65 D. S. Rim, An exact sequence in Galois cohomology, Proc. Amer. Math. Soc. 16 (1965), 837-840.

Rub96 K. Rubin, A Stark conjecture 'Over $\mathbb{Z}$ ' for abelian L-functions with multiple zeros, Ann. Inst. Fourier 46 (1996), 33-62.

Ser79 J.-P. Serre, Local fields, Graduate Texts in Mathematics, vol. 67 (Springer, Berlin, 1979).

Tat84 J. Tate, Les Conjectures de Stark sur les Fonctions L d'Artin en $s=0$, Progress in Mathematics, vol. 47 (Birkhäuser, Basel, 1984).

Tut48 W. T. Tutte, The dissection of equilateral triangles into equilateral triangles, Proc. Camb. Phil. Soc. 44 (1948), 463-482.

Wei67 A. Weil, Basic number theory, Die Grundlehren der mathematischen Wissenschaften, vol. 144 (Springer, New York, 1967).

A. Hayward anthony.hayward@kcl.ac.uk

Department of Mathematics, King's College London, Strand, London WC2R 2LS, UK 